\title{
Nonlinear Elongational Rheology of Unentangled Polystyrene and Poly(p-tert- butylstyrene) Melts
}

Matsumiya, Yumi; Watanabe, Hiroshi; Masubuchi, Yuichi; Huang, Qian; Hassager, Ole

Published in:

Macromolecules

Link to article, DOI:

10.1021/acs.macromol.8b01954

Publication date:

2018

Document Version

Peer reviewed version

Link back to DTU Orbit

Citation (APA):

Matsumiya, Y., Watanabe, H., Masubuchi, Y., Huang, Q., \& Hassager, O. (2018). Nonlinear Elongational Rheology of Unentangled Polystyrene and Poly(p-tert-butylstyrene) Melts. Macromolecules, 51(23), 9710-9729. https://doi.org/10.1021/acs.macromol.8b01954

\section{General rights}

Copyright and moral rights for the publications made accessible in the public portal are retained by the authors and/or other copyright owners and it is a condition of accessing publications that users recognise and abide by the legal requirements associated with these rights.

- Users may download and print one copy of any publication from the public portal for the purpose of private study or research.

- You may not further distribute the material or use it for any profit-making activity or commercial gain

- You may freely distribute the URL identifying the publication in the public portal 


\title{
Nonlinear Elongational Rheology of Unentangled Polystyrene and Poly(p-tert-butyl styrene) Melts
}

\author{
Yumi Matsumiya and Hiroshi Watanabe* \\ Institute for Chemical Research, Kyoto University, Uji, Kyoto 611-0011, Japan
}

Yuichi Masubuchi

Department of Materials Physics, Nagoya University, Furo-cho, Chikusa-ku, Nagoya 4648603, Japan

\author{
Qian Huang and Ole Hassager \\ Department of Chemical and Biochemical Engineering, Technical University of Denmark, \\ Søltofts Plads, 2800 Kgs., Lyngby, Denmark
}

*: to whom all correspondence should be addressed 
Abstract: Nonlinear rheological behavior under uniaxial elongation was examined for unentangled melts of polystyrene (PS27; $M=27 \mathrm{k})$ and poly(p-tert-butyl styrene) (PtBS53; $M=53 \mathrm{k}$ ) having nearly the same number of Kuhn segments per chain, $n_{\mathrm{K}}=30$ and 35 for PS27 and PtBS53, respectively. For both materials, the steady state elongational viscosity $\eta_{\mathrm{E}}$ exhibited strain-rate-hardening and then strain-rate-softening on an increase of the Weissenberg number $W i \geq$ $0.3\left(W i=\dot{\varepsilon} \tau_{1}^{\mathrm{eq}}\right.$, with $\tau_{1}^{\mathrm{eq}}$ and $\dot{\varepsilon}$ being the longest relaxation time in the linear viscoelastic regime and the Hencky strain rate, respectively). For the unentangled melts, the hardening and softening were free from any entanglement nonlinearity, so that the hardening was unequivocally related to the finite extensible nonlinear elasticity (FENE) of the chain, and the softening, to suppression of the FENE effect due to reduction of the segmental friction $\zeta$ occurring for the highly stretched and oriented chain. Thus, the $\zeta$-reduction, speculatively discussed for entangled melts so far, was experimentally confirmed, to the first time, for unentangled melts. Quantitatively, the hardening at intermediate $W i$ was stronger and the softening at higher $W i$ was weaker for PtBS53 than for PS27 despite the similarity of their $n_{\mathrm{K}}$ values, which suggested that the magnitude of $\zeta$-reduction depends on the chemical structure of the chains. For estimation of this magnitude, the FENE-PM model (a FENE bead-spring model with a pre-averaged FENE-spring constant) was modified for the $\zeta$-reduction in an empirical way with an assumption that $\zeta$ at a given time is fully determined by the chain stretch/orientation and thus by the elongational stress $\sigma_{\mathrm{E}}$ at that time. This modified model was able to mimic the steady state $\eta_{\mathrm{E}}$ data excellently, and the $\zeta$-reduction utilized in the modification was weaker for PtBS53 than for PS27k to confirm the dependence of the $\zeta$-reduction on the chemical structure of the chain. Nevertheless, the same modified model failed to mimic the transient stress growth and relaxation data on start-up and cessation of fast flow (at $W i \geq 4$ ), despite its success for the steady state $\eta_{\mathrm{E}}$ data in the entire range of Wi. Specifically, changes of $\zeta$ in the unentangled melts with time during the relaxation for large Wi were delayed compared to the model calculation. This result suggests, as one possibility, that $\zeta$ in those melts is determined not only by the chain stretch/orientation (i.e., by $\sigma_{\mathrm{E}}$ ) at respective times but also by the transient changes of the stretch/orientation (by $\dot{\sigma}_{\mathrm{E}}$ ), with those changes vanishing in the steady state thereby allowing the model to mimic the $\eta_{\mathrm{E}}$ data. The origin of this change of $\zeta$ with the transient changes of the stretch/orientation is discussed in relation to the local motion of the chain necessary for adjusting its friction to the changes of the stretch/orientation environment. 


\section{INTRODUCTION}

Rheological properties of flexible polymers are one of the central subjects in polymer physics. Experimental data so far accumulated ${ }^{1-15}$ indicate that those properties of linear polymers are uniquely determined just by a few parameters such as the chain molecular weight $M$ (and its distribution), the high-frequency glassy modulus $G_{\infty}$, the entanglement plateau modulus $G_{\mathrm{N}}$ and/or the entanglement molecular weight $M_{\mathrm{e}}$ (not relevant for low- $M$ chains), and the friction $\zeta$ of the Rouse segment (stress-sustaining unit in the rubbery relaxation process). For example, for melts of chemically different species such as polystyrene (PS), poly(p-tert-butyl styrene) (PtBS), and poly(vinyl biphenyl) $(\mathrm{PVPh})$, Inoue and coworkers ${ }^{11}$ showed that the complex Young's modulus $E^{*}$ exhibit almost identical $\omega$ dependence separately in the glassy and rubbery relaxation regimes (at high and low angular frequencies $\omega$ ) when normalized by the characteristic moduli, $3 G_{\infty}$ and $3 G_{\mathrm{N}}$ for respective regimes, and plotted against the frequency scaled by respective relaxation times. Nevertheless, for species with a significant chemical difference such as PS and polyisobutylene $(\mathrm{PiB})$, considerable differences were noted for $E^{*}$ in the glassy relaxation regime, possibly because of multiple origins of a glassy component of the stress, e.g., anisotropies in the torsional orientation of individual chains and in the segmental packing. ${ }^{10} \quad$ In contrast, in the rubbery relaxation regime, the $\omega$ dependence of $E^{*}$ is almost identical for chemically different species having the same $M / M_{\mathrm{e}}$ ratio because the stress in this regime has a single origin (axial orientational anisotropy of the Rouse segments), although a delicate difference attributable to a difference in the thermal constraint release time (entanglement loosening time) is still noted for polymers having significantly different chemical structures such as PS and polyisoprene (PI); see Supporting Information of Ref. 12.

This universality in the rubbery relaxation has been most clearly noted for entangled melts and solutions of the same chemical species under shear. For example, for entangled PS melts and solutions having the same $M / M_{\mathrm{e}}$ ratio, Hassager and coworkers ${ }^{13,14}$ showed that the data of the complex shear modulus $G^{*}$ normalized by respective $G_{\mathrm{N}}$ are indistinguishable to each other when plotted against the angular frequency $\omega$ scaled by the relaxation time of the entanglement segment ( $\sim n_{\mathrm{e}}^{2}$ with $n_{\mathrm{e}}$ being the number of Rouse segments per entanglement segment) and that those data are well described by the Baumgaertel-Schausberger-Winter spectrum. ${ }^{7}$ The corresponding universality is noted also under shear flow in the nonlinear regime: $:^{2-6,14}$ The melt and solutions commonly exhibit a stress overshoot peak at a constant strain ( $\dot{\gamma} t \cong 2$ with $\dot{\gamma}$ being the shear rate) 
under relatively slow shear flow, and their steady state shear viscosity $\eta$ normalized by the zero-shear viscosity $\eta_{0}$ exhibits universal thinning behavior as a function of the scaled shear rate $\dot{\gamma} \tau_{1}^{\text {eq }}$, with $\tau_{1}^{\text {eq }}$ being the terminal relaxation time in the linear viscoelastic (LVE) regime. These universal features have encouraged development of molecular models ${ }^{2-6}$ describing the linear and nonlinear rheological behavior of the entangled polymers (in the rubbery relaxation regime) in terms of just a few parameters mentioned above. For example, the tube model for linear polymers, considering reptation, contour length fluctuation (CLF), thermal constraint release (TCR), and convective constraint release (CCR) mechanisms, describes the linear and nonlinear rheological data considerably well, ${ }^{2-6}$ although several problems (such as changes of the CLF path due to TCR ${ }^{12,15}$ ) still remain unsettled.

The universality is also noted for entangled melts and solutions under slow elongational flow in the LVE regime, obviously because $E^{*}=3 G^{*}$ in this regime. However, under fast elongational flow in the nonlinear regime, the universality completely vanishes for the melt and solutions. For example, Sridhar and coworkers ${ }^{16,17}$ found that the steady state elongational viscosity $\eta_{\mathrm{E}}$ of entangled semidilute solutions of linear PS exhibits strain-rate-softening followed by strain-rate-hardening on an increase of the Hencky strain rate $\dot{\varepsilon}$ above the terminal relaxation frequency and further above the Rouse relaxation frequency of the chain as a whole. In contrast, Hassager and coworkers $^{13,14,18-20}$ revealed that $\eta_{\mathrm{E}}$ of entangled linear PS melt just softens (without the hardening) on the corresponding increase of $\dot{\varepsilon}$. This essential difference between the entangled solutions and melts has been attributed to reduction of the segmental friction $\zeta$ occurring in the melt but not in the semidilute solutions..$^{13,14,21-23}$ The $\zeta$-reduction is related to enhanced mobility of a segment in the stretched/oriented environment, as discussed first by Ianniruberto and coworkers ${ }^{21}$ The $\zeta$-reduction can occur in highly stretched melts wherein a segment of a given chain is surrounded by stretched/oriented chains, but not in semidilute solutions because the very rapidly relaxing solvent molecules offer an isotropic environment for the segment. Yaoita and coworkers ${ }^{22}$ examined the stress relaxation data of entangled PS melt from Hassager's group ${ }^{20}$ to find acceleration of the initial relaxation attributable to the stretch/orientation-induced $\zeta$-reduction and the finite-extensible nonlinear elasticity (FENE) effect, estimated the magnitude of $\zeta$-reduction from this acceleration, and demonstrated that the primitive chain network (PCN) simulation with and without the $\zeta$-reduction describes the steady state $\eta_{\mathrm{E}}$ data of PS melt ${ }^{20}$ and solution ${ }^{17}$ considerably well. They 
also suggested that the $\zeta$-reduction can occur also under fast shear but the shear rate necessary to observe this $\zeta$-reduction is too high to be realized in ordinary experiments (because of edge fracture or other instabilities). ${ }^{22}$ Huang, Hassager, and coworkers ${ }^{13,14,23,24}$ examined $\eta_{\mathrm{E}}$ of PS solutions in oligomeric styrene (OS) to demonstrate that the strain-rate-hardening at high $\dot{\varepsilon}$, characteristic to solutions in low- $M$ solvent, is progressively suppressed with increasing molecular weight of OS and that the nematic interaction between PS and OS strongly affects $\eta_{\mathrm{E}}$. Masubuchi and coworkers ${ }^{25}$ examined the elongational data of entangled polyisoprene (PI) and poly(n-butyl acrylate) (PnBA) melts reported by Sridhar and coworkers ${ }^{26}$ with the aid of PCN simulation and compared the results with those for PS melt, thereby suggesting that the magnitude of stretch/orientation-induced $\zeta$-reduction changes with the chemical structure of the chain and has a non-universal character.

Thus, the $\zeta$-reduction is very important in the nonlinear elongational rheology of polymer melts. Nevertheless, for entangled melts so far examined, the elongational behavior should be also affected by the nonlinear mechanisms related to entanglement that include $\mathrm{CCR}^{27}$ and the interchain tube pressure (ICP) proposed by Marrucci and Ianniruberto. ${ }^{28}$ The effect of CCR might be rather minor under the elongational deformation involving no rotational component of motion. In contrast, ICP formulated in the molecular stress function model by Wagner and coworkers ${ }^{29}$ may contribute to the elongational behavior significantly, but the importance of ICP was not found in recent analysis by Desai and Larson. ${ }^{30}$ Interestingly, the tube model phenomenologically modified for the $\zeta$-reduction can mimic the elongational data of entangled melts, as shown by Desai and Larson ${ }^{30}$ and by Masubuchi and coworkers. ${ }^{31}$ Nevertheless, in this modified model, the $\zeta$-reduction is strongly coupled with nonlinearities of entanglement relaxation assumed in the model, so that the details of the $\zeta$-reduction have not been fully elucidated.

Concerning this problem, we note that the $\zeta$-reduction due to the chain stretch and orientation is a local phenomenon essentially irrelevant to the entanglement. (Entanglement just enhances the stretch and orientation.) From this point of view, it is strongly desired to investigate the $\zeta$-reduction under elongational flow for unentangled melts free from the entanglement nonlinearities. Indeed, Masubuchi and coworkers ${ }^{32}$ made the Kremer-Grest-type bead-spring simulation to suggest that the stress relaxation on cessation of elongational flow is accelerated also for unentangled melts but the $\zeta$-reduction underlying this acceleration is a little weaker, for some reason, than that in entangled melts reported in Ref. 22. Thus, it is strongly desired to examine, in the clearest way, the 
$\zeta$-reduction under elongational flow from unambiguous experiments for unentangled melts. To the best of our knowledge, no such experiment has been reported for unentangled $P S$, the most extensively investigated polymer having no specific chemical interaction between the segments. (Very recently, López-Barrón and coworkers examined the elongational behavior of unentangled poly(vinyl biphenyl) melt. ${ }^{33}$ However, the elongational data of this polymer is strongly affected by the $\pi-\pi$ interaction between the segments, and the feature of $\zeta$-reduction cannot be straightforwardly extracted from those data.)

Thus, we have conducted elongational measurements for PS27 melt ( $M=27 \mathrm{k}$, number of Kuhn segments per chain $n_{\mathrm{K}}=30$ ). For comparison, the measurements were made also for PtBS53 melt $\left(M=53 \mathrm{k}, n_{\mathrm{K}}=35\right)$ having nearly the same $n_{\mathrm{K}}$ as PS27. These low- $M$ melts were in the negligibly (or very weakly) entangled state, and are hereafter referred to as unentangled melts for simplicity. It turned out that the unentangled PS27 and PtBS53 melts commonly exhibit strain-rate-hardening of the steady state elongational viscosity $\eta_{\mathrm{E}}$ followed by the strain-rate-softening with increasing strain rate $\dot{\varepsilon}$. The hardening of unentangled melts is unequivocally attributable to the FENE effect (weakened by the $\zeta$-reduction), and the softening, to the $\zeta$-reduction overwhelming the FENE effect. The hardening was weaker and the softening was stronger for PS27 than for PtBS53, demonstrating that the magnitude of $\zeta$-reduction is dependent on the chemical structure of polymers and thus non-universal. (This finding should serve as a firm base for improving the discussion of $\zeta$-reduction in entangled melts explained earlier). Furthermore, the $\eta_{\mathrm{E}}$ data as well as the transient stress data on start-up and cessation of elongational flow were analyzed with the aid of the well established FENE bead-spring model ${ }^{34,35}$ to suggest, as one possibility, that the segmental friction $\zeta$ at a given time depends not only on the chain stretch/orientation at that time but also on the transient changes of the stretch/orientation (being absent in the steady state). These results are presented and discussed in this paper.

\section{EXPERIMENTAL}

\section{2-1. Materials.}

Narrow molecular weight distribution linear polystyrene (PS27), synthesized/characterized by Prof. Atsushi Takano of Nagoya University ${ }^{36}$ and kindly supplied for this study, and linear poly(p-tert-butyl styrene) (PtBS53) synthesized/characterized in our previous study ${ }^{37}$ were utilized. 
Their molecular characteristics are summarized in Table 1. No detectable entanglement effect emerged for the PS27 and PtBS53 samples, as expected from their molecular weights being smaller than the characteristic molecular weight for appearance of the entanglement effect on viscosity, $M_{\mathrm{c}} \cong$ $2 M_{\mathrm{e}}$ (with the entanglement molecular weight $M_{\mathrm{e}}$ being $18{ }_{{ }_{1}} \times 10^{3}$ for $\mathrm{PS}^{38}$ and $37{ }_{6} \times 10^{3}$ for $\mathrm{PtBS}^{38}$ ), and confirmed from their linear viscoelastic data (shown later in Figures 1 and 2).

Table 1. Characteristics of PS and PtBS samples.

\begin{tabular}{|l|c|c|c|}
\hline sample code $^{\mathrm{a}}$ & $10^{-3} M_{\mathrm{w}}$ & $M_{\mathrm{w}} / M_{\mathrm{n}}$ & $n_{\mathrm{K}}{ }^{\mathrm{b}}$ \\
\hline PS27 & 27.1 & 1.02 & 30 \\
\hline PtBS53 $^{\mathrm{d}}$ & 53.4 & 1.03 & 35 \\
\hline
\end{tabular}

a: sample code indicates molecular weight in unit of 1000 .

b: number of Kuhn segments per chain

c: synthesized/characterized in ref. 36; PS27 is a precursor of a tadpole sample examined therein.

d: synthesized/characterized in ref. 37

The number of Kuhn segments per chain, $n_{\mathrm{K}}=M / M_{\mathrm{K}}$ shown in Table 1, was calculated from the chain molecular weight $M\left(M_{\mathrm{w}}\right)$ and the molecular weight of the Kuhn segment $M_{\mathrm{K}}: M_{\mathrm{K}} \cong 900$ for PS and $M_{\mathrm{K}} \cong 1500$ for PtBS. ${ }^{39} \quad$ These $M_{\mathrm{K}}$ values, characterizing the chain conformation, are very close to the molecular weight of the Rouse segment that serves as the smallest stress-sustaining unit in the rubbery relaxation process: $M_{\mathrm{R}} \cong 850$ and 1500 for PS and PtBS, as obtained from rheo-optical analysis by Inoue and coworkers. ${ }^{40}$ Thus, the number of Rouse segments per chain, $n_{\mathrm{R}}=M / M_{\mathrm{R}}$, is almost indistinguishable from $n_{\mathrm{K}}$.

The PS27 and PtBS53 samples have similar $n_{\mathrm{K}}$ values and thus their maximum chain stretch ratios, $\lambda_{\max }=\sqrt{n_{\mathrm{K}}}(\cong 6)$, are very close to each other. In fact, this study chose these samples with a specific motivation of testing the elongational behavior of chemically different unentangled melts that were supposed to exhibit the FENE effect to a similar magnitude (as determined by $\lambda_{\max }$ ) but possibly show different magnitudes of the stretch/orientation-induced reduction of the friction $\zeta$ of the Rouse segment.

\section{2-2. Measurement.}

The PS27 and PtBS53 samples were vacuum-molded into disks at temperatures well above their glass transition temperatures $T_{\mathrm{g}}$ and subjected to rheological measurements. The disk diameter, either $7.9 \mathrm{~mm}$ or $5.0 \mathrm{~mm}$, matched the measuring geometry explained below. After each measurement, lack of degradation of the samples was confirmed from measurements of refractive 
index increment and low-angle laser light scattering intensity utilizing RI-8020 (Tosoh) and Viscotek 270 (Malvern) connected in series to GPC instrument (CO-8020 and DP-8020; Tosoh).

For the PS27 and PtBS53 samples, linear viscoelastic (LVE) oscillatory shear measurement was conducted with a laboratory rheometer, ARES-G2 (TA Instruments), at several temperatures above $T_{\mathrm{g}}$. A cone-and-plate fixture of the diameter of $7.9 \mathrm{~mm}$ and the gap angle of $0.1 \mathrm{rad}$ was used. The data of the storage and loss moduli, $G^{\prime}(\omega)$ and $G^{\prime \prime}(\omega)$, were summarized as the master curve reduced at a reference temperature, $T_{\mathrm{r}}=115^{\circ} \mathrm{C}$ for PS27 and $T_{\mathrm{r}}=170^{\circ} \mathrm{C}$ for PtBS53, to confirm lack of entanglement for the PS27 and PtBS53 samples. Shear flow experiments were also conducted at those $T_{\mathrm{r}}$ to measure the shear stress growth coefficient $\eta^{+}(t)$ and the zero-shear viscosity $\eta_{0}(=$ $\left.\left[\eta^{+}\right]_{t \rightarrow \infty}\right)$ in the LVE regime. This $\eta^{+}(t)$ was utilized as the reference data for the elongational stress growth explained below.

For the PS27 and PtBS53 samples at respective $T_{\mathrm{r}}$ mentioned above, elongational measurements were conducted with a filament-stretch rheometer, VADER1000 (Rheo Filament ApS; developed and commercialized by Hassager's group). The parallel plate geometry of the diameter of $6 \mathrm{~mm}$ was used. The samples, pre-molded in vacuum into disks of a diameter of $5 \mathrm{~mm}$, were gripped by the plates and uniaxially stretched into filaments at constant Hencky strain rates $\dot{\varepsilon}$ up to the maximum strain of $\varepsilon=2.5$ or higher (up to the sample rupture). The relaxation measurement was also conducted by stopping the flow at a pre-set strain, $\varepsilon=2.5$. The constant rate elongation (for the stress growth) and time-independent strain (for the stress relaxation) were achieved through measurement of the filament diameter and feedback to the plate displacement, both being automated in VADER 1000.

The tensile stress $\sigma_{\mathrm{E}}$ measured on start-up of the constant-rate flow, in the steady flow state, and after cessation of the flow at $\varepsilon=2.5$, respectively, were summarized as the elongational stress growth coefficient $\eta_{\mathrm{E}}^{+}(t, \dot{\varepsilon}) \quad\left(=\sigma_{\mathrm{E}}^{+}(t, \dot{\varepsilon}) / \dot{\varepsilon}\right)$, the steady state elongational viscosity $\eta_{\mathrm{E}}(\dot{\varepsilon})$, and the relaxing stress ratio $\sigma_{\mathrm{E}}^{-}(t) / \sigma_{\mathrm{E}}^{-}\left(t_{\mathrm{r}}\right)$ with $t_{\mathrm{r}}(=0.4 \mathrm{~s})$ being a time required for accurate feedback of the plate position for keeping the constant strain $(\varepsilon=2.5)$ after the flow cessation.

\section{RESULTS}

\section{3-1. Linear Viscoelastic Behavior.}

Figures 1 and 2, respectively, show master curves of the $G^{\prime}(\omega)$ and $G^{\prime \prime}(\omega)$ data of PS27 and 
PtBS 53 reduced at $T_{\mathrm{r}}=115^{\circ} \mathrm{C}$ and $170^{\circ} \mathrm{C}$, respectively. Data measured at different temperatures are shown with different symbols (see Figure caption), and the shift factor $a_{T}$ utilized to construct the master curves is shown in inset.

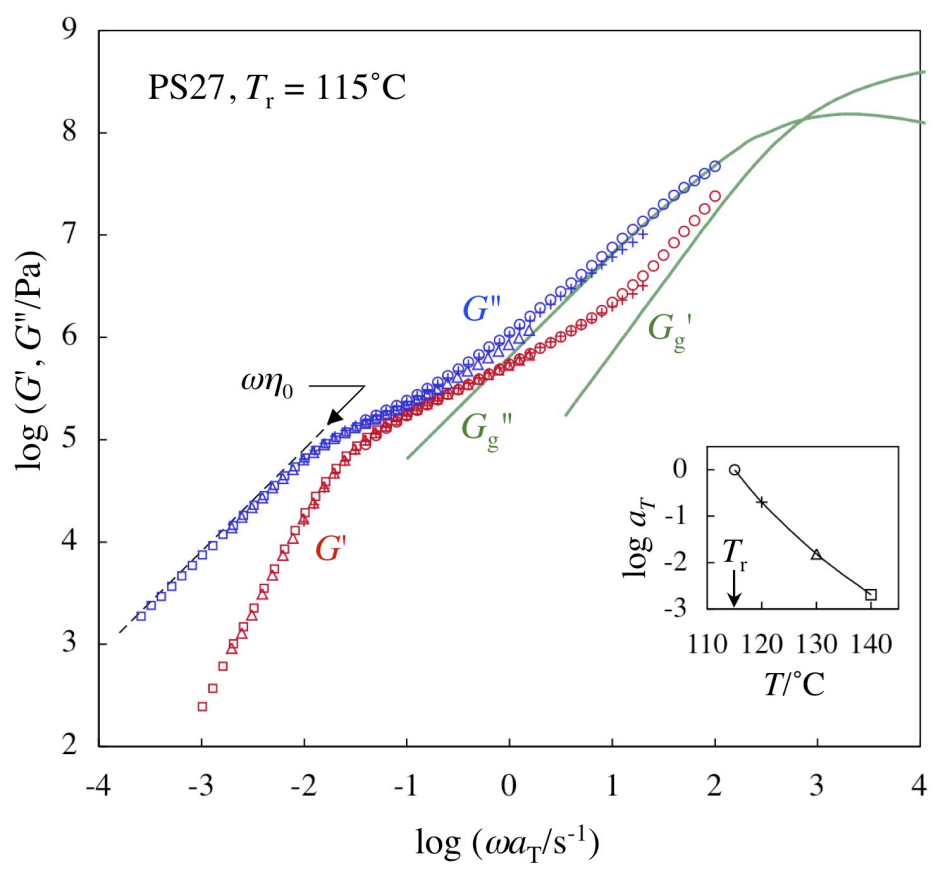

Figure 1. Storage and loss moduli of PS27 reduced at $T_{\mathrm{r}}=115^{\circ} \mathrm{C}$. The data measured at different temperatures are shown with different symbols, circle $\left(115^{\circ} \mathrm{C}=T_{\mathrm{r}}\right)$, plus $\left(120^{\circ} \mathrm{C}\right)$, triangle $\left(130^{\circ} \mathrm{C}\right)$, and square $\left(130^{\circ} \mathrm{C}\right)$, and the shift factor $a_{T}$ for those data is shown in the inset. Green curves indicate glassy contribution to the modulus evaluated from literature data. ${ }^{9}$

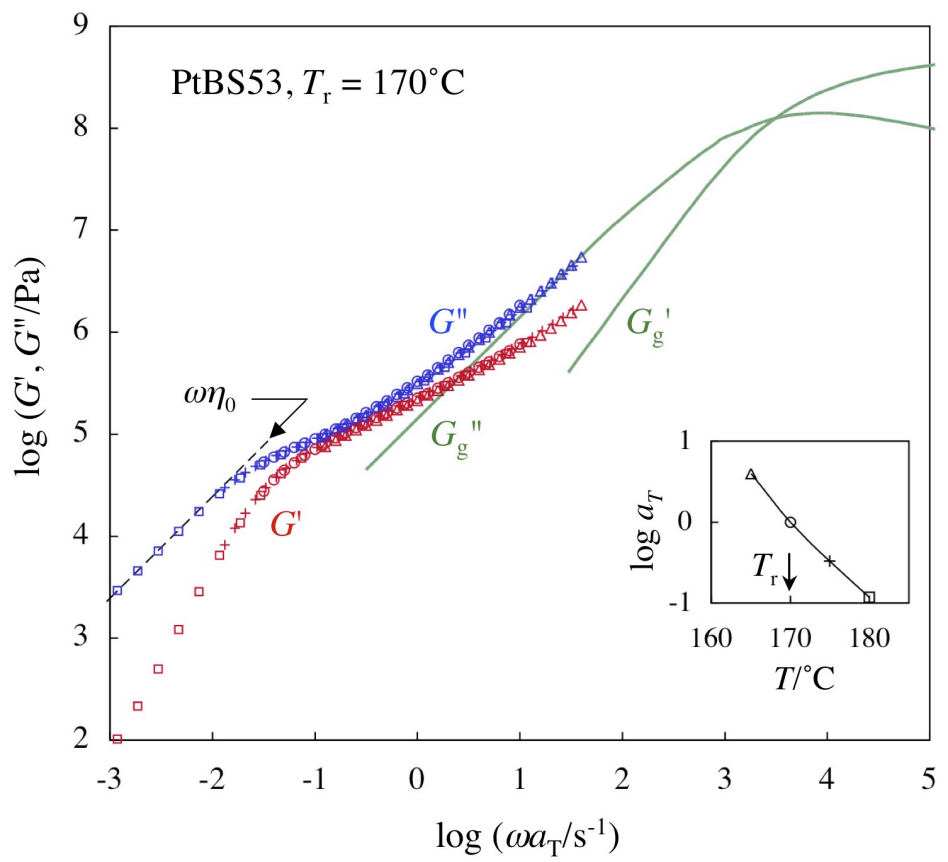

Figure 2. Storage and loss moduli of PtBS53 reduced at $T_{\mathrm{r}}=170^{\circ} \mathrm{C}$. The data measured at different temperatures are shown with different symbols, triangle $\left(165^{\circ} \mathrm{C}\right)$, circle $\left(170^{\circ} \mathrm{C}=T_{\mathrm{r}}\right)$, plus $\left(175^{\circ} \mathrm{C}\right)$, and square $\left(180^{\circ} \mathrm{C}\right)$, and the shift factor $a_{T}$ for those data is shown in the inset. Green curves indicate glassy contribution to the modulus evaluated from literature data. ${ }^{11}$ 
As noted in Figures 1 and 2, the time-temperature superposition (tTS) does not work well in the rubber-to-glass transition regime (as can be noted more clearly for PS27 because of a wider range of $\omega$ covered for PS27), because the glassy and rubbery relaxation processes having different activation energies contribute to the data at high $\omega$ to a comparable extent, as demonstrated by Inoue and coworkers. ${ }^{9-11,40}$ Nevertheless, the unentangled Rouse-like character of PS27 and PtBS53 (having $M<M_{\mathrm{c}} \cong 2 M_{\mathrm{e}}$ ) is clearly noted at low $\omega$ where the data are dominantly contributed from the rubbery relaxation process and tTS works well. The terminal relaxation time of the samples are evaluated from those data as

$$
\langle\tau\rangle=\left[\frac{G^{\prime}}{\omega G^{\prime \prime}}\right]_{\omega \rightarrow 0}=30 \mathrm{~s}\left(\text { for } \operatorname{PS} 27 \text { at } 115^{\circ} \mathrm{C}\right), 28 \mathrm{~s}\left(\text { for PtBS53 at } 170^{\circ} \mathrm{C}\right)
$$

This $\langle\tau\rangle$, being defined as the second-moment average relaxation time ${ }^{2,5}\left(=\sum_{p} h_{p} \tau_{p}^{2} / \Sigma_{p} h_{p} \tau_{p}\right.$ with $h_{p}$ and $\tau_{p}$ being the intensity and characteristic time of $p$-th viscoelastic relaxation mode), is a little shorter than the longest relaxation time $\tau_{1}^{\text {eq }}$ evaluated from the stress relaxation data after cessation of flow in the LVE regime (shown later in Figures 6 and 7),

$$
\tau_{1}^{\mathrm{eq}}=43 \mathrm{~s}\left(\text { for } \mathrm{PS} 27 \text { at } 115^{\circ} \mathrm{C}\right), \quad 40 \mathrm{~s}\left(\text { for PtBS53 at } 170^{\circ} \mathrm{C}\right)
$$

The $\langle\tau\rangle / \tau_{1}^{\mathrm{eq}}$ ratios of PS27 and PtBS53 are close to the ratio expected for Rouse chains ${ }^{5}\left(=\pi^{2} / 15\right.$ in the absence of molecular weight distribution), confirming the unentangled character of these samples. The $\tau_{1}^{\mathrm{eq}}$ values (eq 2) are later utilized to evaluate the Weissenberg number of elongational flow.

Inoue and coworkers conducted rheo-optical measurements for high- $M$ PS ${ }^{9}$ and high- $M$ PtBS ${ }^{11}$ to separate the complex Young's modulus $\left(E^{*}\right)$ data into glassy and rubber contributions with the aid of the modified stress-optical rule. This glassy contribution, being converted to the shear modulus $G_{\mathrm{g}}{ }^{*}(\omega)=E_{\mathrm{g}}{ }^{*}(\omega) / 3$ and shifted to $T_{\mathrm{r}}$ of our PS27 and PtBS53 (with the aid of the $a_{\mathrm{T}}$ data for the glassy $E_{\mathrm{g}}{ }^{*}$ reported by Inoue and coworkers), is shown in Figures 1 and 2 with the green curves. The data of PS27 and PtBS53 approach this glassy modulus with increasing $\omega$, which allows us to estimate the characteristic time of the glassy relaxation $\tau_{\mathrm{g}}$ of these samples at $T_{\mathrm{r}}$ as reciprocal of the angular frequency at the crossing point of the $G_{\mathrm{g}}{ }^{\prime}$ and $G_{\mathrm{g}}{ }^{\prime \prime}$ curves:

$$
\tau_{\mathrm{g}}=1.3 \times 10^{-3} \mathrm{~s}\left(\text { for PS27 at } 115^{\circ} \mathrm{C}\right), \quad 0.32 \times 10^{-3} \mathrm{~s}\left(\operatorname{PtBS} 53 \text { at } 170^{\circ} \mathrm{C}\right)
$$

These $\tau_{\mathrm{g}}$ values are utilized later in discussion of the elongational behavior. 
In Figures 1 and 2, black dashed lines indicate the low- $\omega$ asymptote of $G^{\prime \prime}\left(=\omega \eta_{0}\right)$, with the zero-shear viscosity $\eta_{0}$ being determined directly from the steady shear experiments at $\dot{\gamma}=0.01 \mathrm{~s}^{-1}$ (in the LVE regime). This asymptote agrees well with the $G^{\prime \prime}$ data at low $\omega$, as required from the phenomenological framework of LVE. Comparing these black dashed lines with the green curves at low $\omega$, we note that the glassy contribution (green curves) to $\eta_{0}$ of the PS27 and PtBS53 samples is $\sim 5 \%$. This glassy contribution is later incorporated in the calculation of the elongational viscosity based on the bead-spring model considering the FENE effect (FENE-PM model). ${ }^{34}$

\section{3-2. Nonlinear Elongational Behavior.}

In Figures 3 and 4, respectively, the elongational stress growth coefficient $\eta_{E}^{+}(t, \dot{\varepsilon})$ measured for PS27 and PtBS53 at various Hencky strain rates $\dot{\varepsilon}$ is plotted double-logarithmically against the time $t$ after start-up of flow; see unfilled circles with the color specifying the $\dot{\varepsilon}$ value. The flow was not well stabilized immediately after the start-up, mainly because of limitation of the feedback in the instrument for starting the plate displacement from the quiescent state. Thus, the plots of the $\eta_{E}^{+}(t, \dot{\varepsilon})$ data are shown at $t>1 \mathrm{~s}$ where this feedback worked well. Bright green squares indicate the growth coefficient $\eta_{\mathrm{E} 0}^{+}(t) \equiv 3 \eta^{+}(t)$ in the LVE regime, with $\eta^{+}(t)$ being the shear stress growth coefficient measured at $\dot{\gamma}=0.01 \mathrm{~s}^{-1}$. At sufficiently short $t$ where the strain remains small and no significant nonlinearity emerges, all data points (circles and squares) agree with each other within the experimental uncertainty. This feature is indicative of satisfactory accuracy of those data.

In Figures 3 and 4, the colored solid curves and asterisks $(*)$ indicate $\eta_{\mathrm{E}}^{+}$calculated from FENE-PM model ${ }^{32}$ modified for the $\zeta$-reduction, as explained later in detail. The thick black curve shows $\eta_{\mathrm{E} 0}^{+}(t)$ in the LVE regime calculated from that model. This black curve agrees with the measured $\eta_{\mathrm{E} 0}^{+}(t)$ (bright green squares) in the entire range of $t$ within the experimental uncertainty. We also note that all colored curves converge to the black LVE curve at sufficiently short $t$ where the strain remains small and no significant nonlinearity emerges in the calculation, as similar to the behavior of the data. 


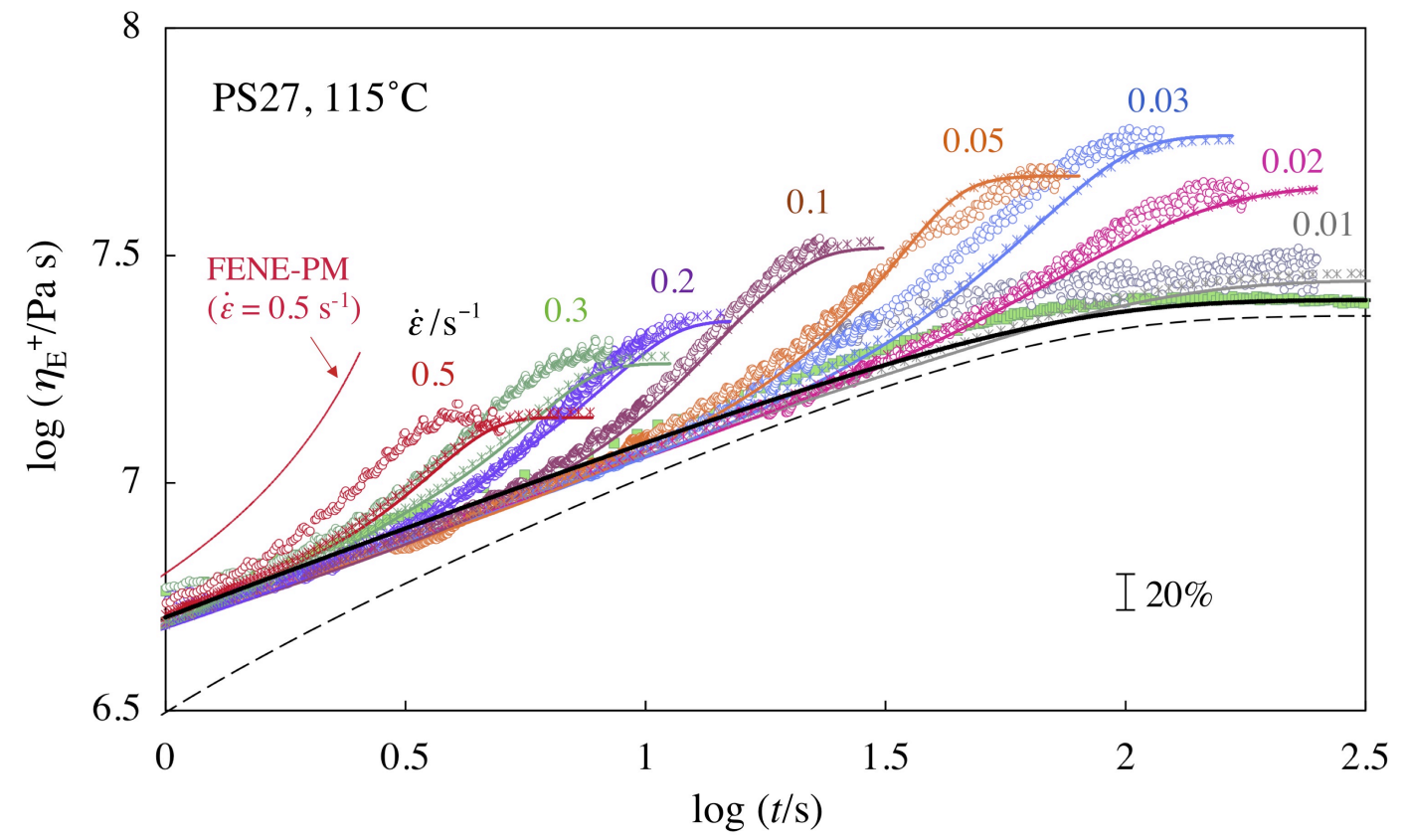

Figure 3. Elongational stress growth coefficient $\eta_{\mathrm{E}}^{+}(t, \dot{\varepsilon})$ of PS27 at $115^{\circ} \mathrm{C}$ (colored circles). Bright green squares show the linear viscoelastic elongational behavior, $\eta_{\mathrm{E} 0}^{+}(t) \equiv 3 \eta^{+}(t)$ with $\eta^{+}(t)$ being the shear stress growth coefficient of PS27 in the LVE regime measured at $115^{\circ} \mathrm{C}$. Colored asterisks $(*)$ and curves show $\eta_{\mathrm{E}}^{+}(t, \dot{\varepsilon})$ calculated from FENE-PM model modified for anisotropic and isotropic $\zeta$-reduction, respectively. Black solid and dashed curves, respectively, show the FENE-PM calculation in LVE regime with and without the glassy contribution. For further details of calculation, see text.

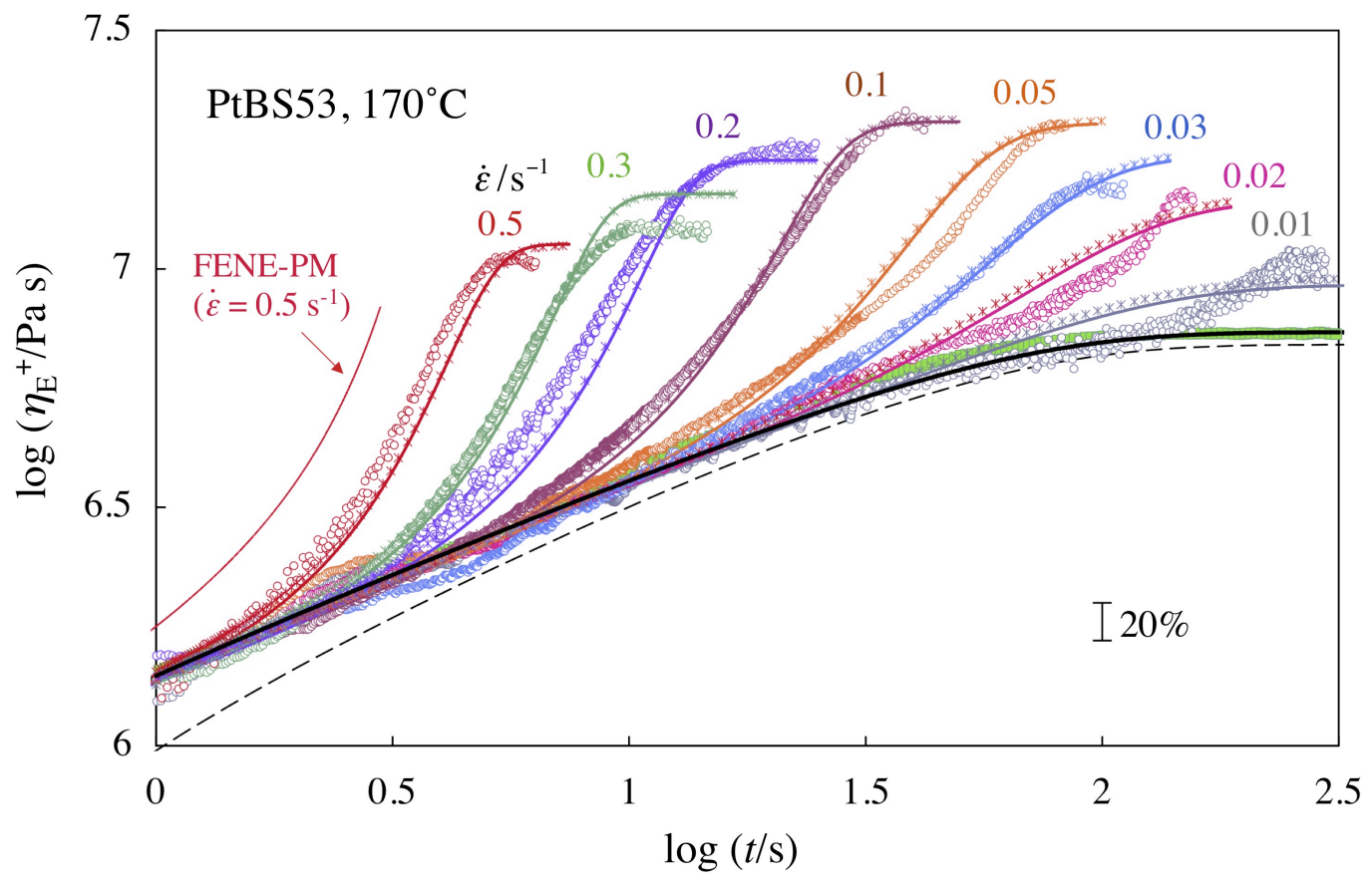

Figure 4. Elongational stress growth coefficient $\eta_{\mathrm{E}}^{+}(t, \dot{\varepsilon})$ of PtBS53 at $170^{\circ} \mathrm{C}$ (colored circles). Bright green squares show the linear viscoelastic elongational behavior, $\eta_{\mathrm{E} 0}^{+}(t) \equiv 3 \eta^{+}(t)$ with $\eta^{+}(t)$ being the shear stress growth coefficient of PtBS53 in the LVE regime measured at $170^{\circ} \mathrm{C}$. Colored asterisks (*) and curves show $\eta_{\mathrm{E}}^{+}(t, \dot{\varepsilon})$ calculated from FENE-PM model modified for anisotropic and isotropic $\zeta$-reduction, respectively. Black solid and dashed curves, respectively, show the FENE-PM calculation in LVE regime with and without the glassy contribution. For further details of calculation, see text. 
For both PS27 and PtBS53, the $\eta_{E}^{+}(t, \dot{\varepsilon})$ data at low $\dot{\varepsilon}=0.01 \mathrm{~s}^{-1}$ (gray circles in Figures 3 and 4) are close to the $\eta_{\mathrm{E} 0}^{+}(t)$ data (bright green squares) in the entire range of $t$, confirming that the elongational behavior at such low $\dot{\varepsilon}\left(\cong 0.4 / \tau_{1}^{\text {eq }}\right.$ with $\tau_{1}^{\text {eq }}$ being the longest relaxation time in the LVE regime; cf. eq 2) is not significantly different from the LVE behavior. This almost linear response vanishes and the transient hardening behavior prevails at higher $\dot{\varepsilon}$. Those $\eta_{E}^{+}(t, \dot{\varepsilon})$ data become stationary at long $t$, and the steady state elongational viscosity $\eta_{\mathrm{E}}(\dot{\varepsilon})$ is evaluated as $\eta_{E}^{+}(t, \dot{\varepsilon})$ at such long $t$.

In Figure 5, this $\eta_{\mathrm{E}}(\dot{\varepsilon})$ is normalized by the "zero-elongation" viscosity $\eta_{\mathrm{E} 0}=3 \eta_{0}$ (with $\eta_{0}$ being the zero-shear viscosity explained for Figures 1 and 2) and double-logarithmically plotted against the Weissenberg number (normalized strain rate) $W i=\dot{\varepsilon} \tau_{1}^{\text {eq }}$ defined with respect to $\tau_{1}^{\text {eq }}$ (eq 2); see large blue circles. The curves and small circles indicate the $\eta_{\mathrm{E}} / \eta_{\mathrm{E} 0}$ ratio calculated with the FENE-PM mode ${ }^{34}$ modified for $\zeta$-reduction, as explained later in detail.

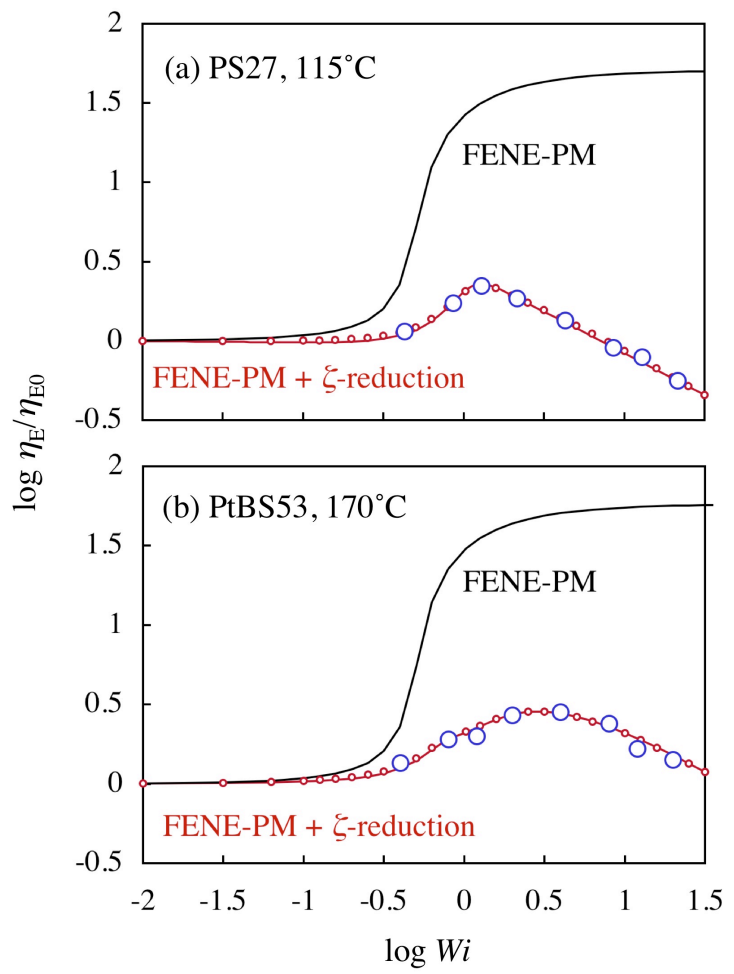

Figure 5. Steady state elongational viscosity $\eta_{\mathrm{E}}$ of (a) PS27 at $115^{\circ} \mathrm{C}$ and (b) PtBS53 at $170^{\circ} \mathrm{C}$. The $\eta_{\mathrm{E}}$ data are normalized by $\eta_{\mathrm{E} 0}=3 \eta_{0}\left(\eta_{0}=\right.$ zero-shear viscosity $)$ and plotted against the Weissenberg number $W i=\dot{\varepsilon} \tau_{1}^{\text {eq }}$, with $\tau_{1}^{\text {eq }}$ being the longest relaxation time in the LVE regime measured after cessation of elongational flow. The small red circle and red curve indicate the $\eta_{\mathrm{E}} / \eta_{\mathrm{E} 0}$ ratio calculated from FENE-PM model modified for anisotropic and isotropic $\zeta$-reduction, respectively. Black curve shows the FENE-PM calculation without $\zeta$-reduction. For further details of calculation, see text. 
As clearly noted in Figure 5, both PS27 and PtBS samples exhibit an increase of the $\eta_{\mathrm{E}} / \eta_{\mathrm{E} 0}$ ratio (strain-rate-hardening) on an increase of $W i$ from $\sim 0.4$ up to 2-3 and then a decrease of this ratio (strain-rate-softening) on a further increase of Wi. For the unentangled PS27 and PtBS53 chains, the longest relaxation time $\tau_{1}^{\mathrm{eq}}$ corresponds to the Rouse motion of the chain as a whole. For such unentangled chains, the established molecular theories ${ }^{3,34,35,41-43}$ suggest that the hardening under elongational flow emerging at $W i \sim 0.5$ is almost exclusively related to the FENE effect due to the chain stretch, and experiments ${ }^{3,41-43}$ (mostly conducted for dilute/semidilute solutions) support this assignment. Consequently, the softening (not occurring in those solutions) can be related to the $\zeta$-reduction in melts that tends to suppress the chain stretch. Thus, the hardening seen for the PS27 and PtBS53 samples is attributed to the FENE effect weakened by the $\zeta$-reduction of the Rouse segment, and the softening, to the $\zeta$-reduction that overwhelms the FENE effect at high Wi. It should be emphasized that lack of entanglement nonlinearities in the PS27 and PtBS53 samples allows us to make this simple assignment without any ambiguity. (For entangled chains, the longest viscoelastic relaxation time $\tau_{1}^{\mathrm{eq}}$ is longer than the Rouse relaxation time of the chain and thus the entanglement nonlinearity is always superimposed on the nonlinearities due to the FENE effect and $\zeta$-reduction, which often disturbs simple experimental assignment of the latter nonlinearities.)

Both FENE effect and $\zeta$-reduction accelerate the stress relaxation just after cessation of elongational flow, as demonstrated by Yaoita and coworkers ${ }^{22}$ (for entangled PS). We examined if this acceleration occurs also for our unentangled melts after cessation of the flow at the strain $\varepsilon=$ 2.5. The results are summarized in Figures 6 and 7 where the relaxing tensile stress $\sigma_{\mathrm{E}}^{-}(t)$, normalized by the stress $\sigma_{\mathrm{E}}^{-}\left(t_{\mathrm{r}}\right)$ at a reference time $t_{\mathrm{r}}=0.4 \mathrm{~s}$, is semi-logarithmically plotted against the time after cessation of the flow; see circles with the color specifying the $\dot{\varepsilon}$ value of the flow. (In the relaxation experiment, the plate displacement at $t<0$ is stopped once and then immediately re-started at $t=0$ to keep the constant strain $(\varepsilon=2.5)$ at $t>0$. Because of the instrumental limitation in the feedback for this re-start, the data were accurately measured only at $t$ $\geq t_{\mathrm{r}}$.) The asterisks $(*)$ and curves indicate the $\sigma_{\mathrm{E}}^{-}(t) / \sigma_{\mathrm{E}}^{-}\left(t_{\mathrm{r}}\right)$ ratio calculated from FENE-PM model ${ }^{34}$ modified for the $\zeta$-reduction, as explained later in detail. 


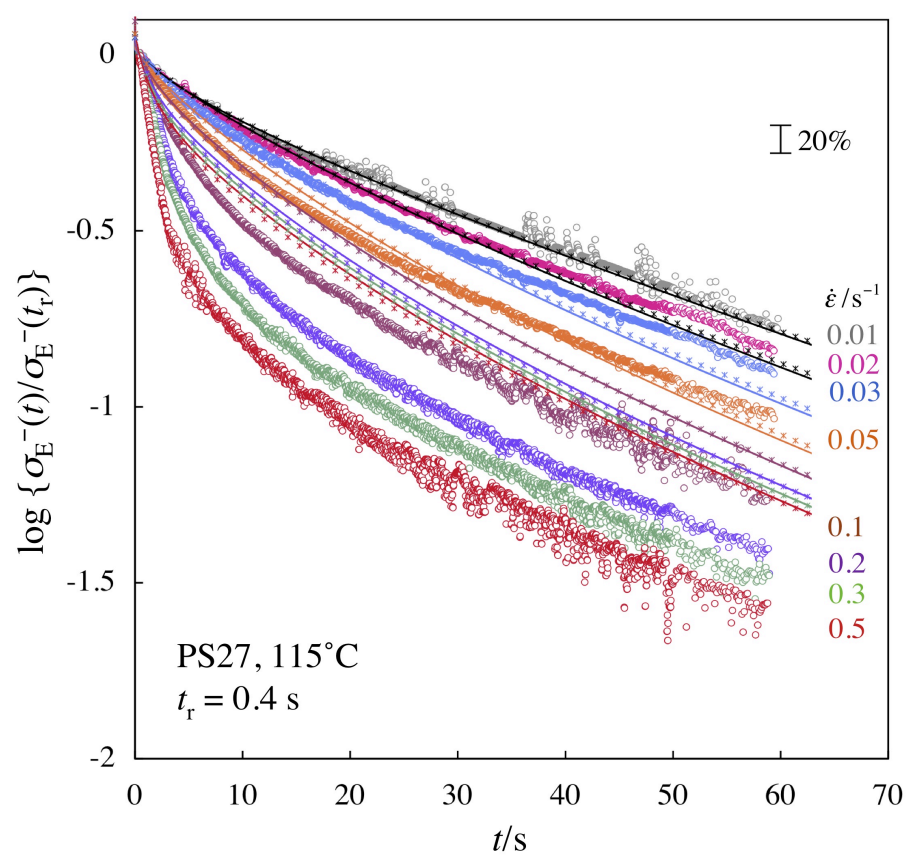

Figure 6. Tensile stress $\sigma_{\mathrm{E}}^{-}(t)$ after cessation of flow at $\varepsilon=2.5$ measured for PS27 at $115^{\circ} \mathrm{C}$. The $\sigma_{\mathrm{E}}^{-}(t)$ data are normalized by the stress at the reference time during the relaxation, $t_{\mathrm{r}}=0.4 \mathrm{~s}$, and plotted semi-logarithmically against $t$. Asterisks $(*)$ and curves indicate the $\sigma_{\mathrm{E}}^{-}(t) / \sigma_{\mathrm{E}}^{-}\left(t_{\mathrm{r}}\right)$ ratio calculated from the FENE-PM model modified for anisotropic and isotropic $\zeta$-reduction, respectively. The color of the asterisk and curve is the same as that of data (circle), except for the black asterisks and curves that are overlapping with the data (and not clearly visible if they have the same color as the data). For further details of calculation, see text.

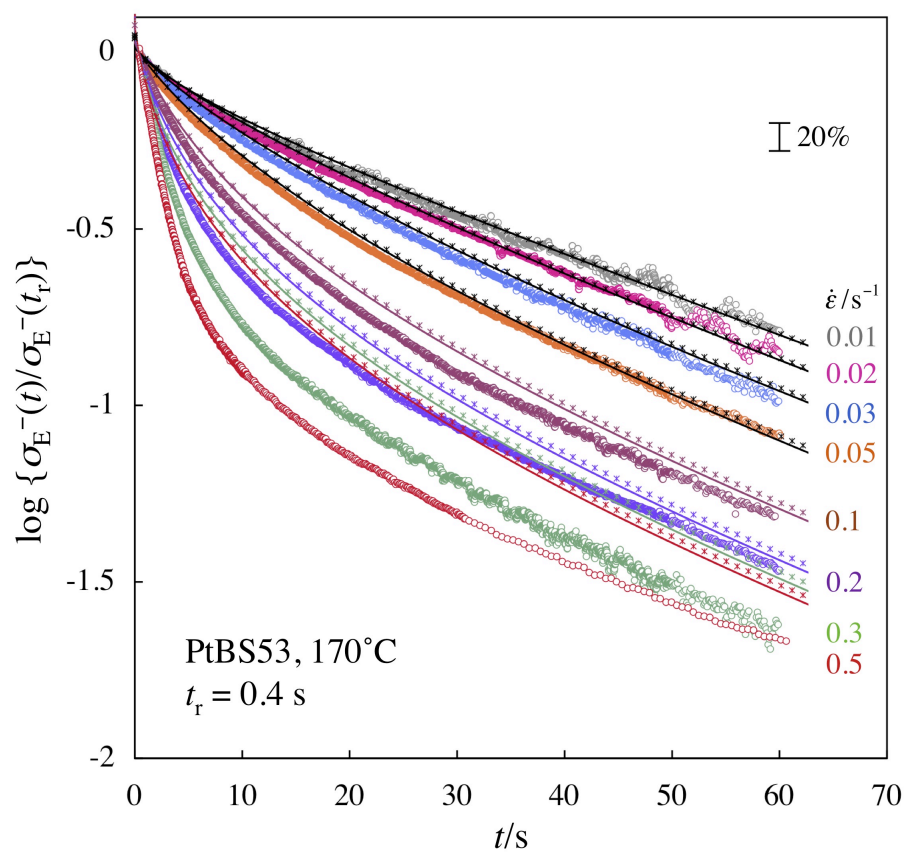

Figure 7. Tensile stress $\sigma_{\mathrm{E}}^{-}(t)$ after cessation of flow at $\varepsilon=2.5$ measured for PtBS53 at $170^{\circ} \mathrm{C}$. The $\sigma_{\mathrm{E}}^{-}(t)$ data are normalized by the stress at the reference time during the relaxation, $t_{\mathrm{r}}=0.4 \mathrm{~s}$, and plotted semi-logarithmically against $t$. Asterisks $(*)$ and curves indicate the $\sigma_{\mathrm{E}}^{-}(t) / \sigma_{\mathrm{E}}^{-}\left(t_{\mathrm{r}}\right)$ ratio calculated from the FENE-PM model modified for anisotropic and isotropic $\zeta$-reduction, respectively. The color of the asterisk and curve is the same as that of data (circle), except for the black asterisks and curves that are overlapping with the data (and not clearly visible if they have the same color as the data). For further details of calculation, see text. 
As clearly noted in Figures 6 and 7, the stress relaxation just after the flow cessation is accelerated and this acceleration is stronger for cessation of faster flow, which confirms the effects of FENE and $\xi$-reduction on the initial relaxation. The strong, initial acceleration just after cessation of fast flow appears to be mostly due to the $\zeta$-reduction, as suggested from a model analysis explained later. For example, for the highest $\dot{\varepsilon}\left(=0.5 \mathrm{~s}^{-1}\right)$ examined, the initial acceleration due to the $\zeta$-reduction deduced from the analysis is stronger than that due to the FENE effect by a factor of $\sim 30$ and $\sim 20$ for PS27 and PtBS53, respectively, as shown later in Figure 17.

In Figures 6 and 7, we also note that the relaxation rate at long $t\left(>\tau_{1}^{\mathrm{eq}} \cong 40 \mathrm{~s}\right)$ becomes independent of $\dot{\varepsilon}$ because these effects due to the chain stretch fade out on relaxation of the stretch. From the slope of the $\sigma_{\mathrm{E}}^{-}(t) / \sigma_{\mathrm{E}}^{-}\left(t_{\mathrm{r}}\right)$ plots at such long $t$, the longest relaxation time $\tau_{1}^{\mathrm{eq}}$ being insensitive to $\dot{\varepsilon}$ (and identical to that in the LVE regime) was evaluated. The $\tau_{1}^{\text {eq }}$ values thus obtained for PS27 and PtBS53 have been summarized in eq 2.

Here, it is informative to compare the nonlinearities observed for PS27 and PtBS53 samples. These samples have a similar number of Kuhn segments per chain, $n_{\mathrm{K}}$ (cf. Table 1), and a very similar value of the maximum stretch ratio of the chain, $\lambda_{\max }=\sqrt{n_{\mathrm{K}}}(\cong 6)$. Thus, the magnitude of the FENE effect determined by this $\lambda_{\max }$ should be similar for the PS27 and PtBS53 chains. Nevertheless, on start-up of flow and in the steady state, the hardening at intermediate Wi is weaker and the softening at higher Wi is stronger for PS27 than for PtBS53, as clearly noted in Figures 3-5. (The maximum $\eta_{\mathrm{E}} / \eta_{\mathrm{E} 0}$ ratio observed for PS27 and PtBS53 is $\sim 2$ and $\sim 3$; cf. Figure 5.) This fact strongly suggests that the $\zeta$-reduction (of the Rouse segment) at large Wi is stronger for PS27 thereby giving a smaller $\eta_{\mathrm{E}} / \eta_{\mathrm{E} 0}$ ratio (Figure 5) and a smaller $\eta_{\mathrm{E}}^{+}(t, \dot{\varepsilon}) / \eta_{\mathrm{E} 0}^{+}(t)$ ratio (Figures 3 and 4) as compared to PtBS53. The corresponding difference in the stress relaxation is not so clearly noted in Figures 6 and 7 but is confirmed in the analysis presented in the following sections.

The above observation strongly suggests that the magnitude of $\zeta$-reduction is dependent on the chemical structure of the chain. The side group of the PtBS chain, p-tert-butyl phenyl group, is much more bulky compared to the phenyl group of the PS chain, which would have resulted in screening of the interaction between the chain backbones to disturb the local packing of the chain segments. In other words, the bulky side group of PtBS appears to behave more or less similar to a solvent thereby suppressing the $\zeta$-reduction. This argument is consistent with the observation for entangled PS solutions in oligomeric solvents ${ }^{13,14,44}$ and with the difference noted between 
entangled melts of PS and PnBA (the $\zeta$-reduction being weaker for the latter having a larger side group). ${ }^{25}$

In summary from a purely experimental point of view, the data presented in Figures 3-7 are not infected by the entanglement nonlinearities and thus have allowed us to confirm, to the first time, the FENE effect and $\zeta$-reduction for unentangled melts as well as the non-universality of $\zeta$-reduction (changes of the magnitude of $\zeta$-reduction with the chemical structure of the chain). These results should serve as a firm base for improving the discussion of $\zeta$-reduction in entangled melts (and of the non-universality of the relaxation therein) explained in Introduction.

However, the above experimental results themselves do not allow us to resolve quantitative details of $\zeta$-reduction, for example, a quantitative difference in the magnitudes of $\zeta$-reduction in PS27 and PtBS53 melts. For investigation of such details, we need to utilize a reliable molecular model describing the FENE effect and modify it for $\zeta$-reduction. This modification and the corresponding model calculation are presented in the following DISCUSSION section.

\section{DISCUSSION}

For the unentangled PS27 and PtBS53 melts, we may start with a FENE bead-spring model, modify it for $\zeta$-reduction, and analyze the data with the aid of this modified model to extract quantitative details of $\xi$-reduction. There are several FENE bead-spring models that treat the

FENE spring stiffness differently. ${ }^{34,35,45}$ Among those models, this study adopts the FENE-PM bead-spring model formulated by Wedgewood, Ostrov, and Bird ${ }^{34}$ for the following reasons. This model includes an approximation of pre-averaging the FENE spring strength for all springs in the chain. However, its prediction for the tensile stress in the steady state is close to that obtained from the more rigorous FENE-P model without this pre-averaging. ${ }^{34}$ More importantly for our current purpose, the FENE-PM model allows the orientation function of the chain to be decomposed into eigenmodes because of the pre-averaging approximation (whereas the FENE-P model does not), and the FENE-PM constitutive equations for the stress of respective eigenmodes explicitly include the segmental friction $\zeta$ so that the $\zeta$-reduction can be easily incorporated in those equations.

Thus, in the followings, we modify the FENE-PM constitutive equation for the $\zeta$-reduction and analyze the modified equation, firstly for the steady elongational flow and then for start-up and cessation of the flow, to examine if the modified FENE-PM model can consistently mimic the data 
of the unentangled melts in the steady and transient states shown in Figures 3-7. It should be emphasized that we are attempting to extract, through this analysis, some quantitative details of $\zeta$-reduction such as the relative ratio of the magnitude of $\xi$-reduction in the PS27 and PtBS53 melts. No attempt is made for formulating a complete molecular model that fully describes the data. Obviously, our analysis starts with a particular model (FENE-PM model) and does not necessarily resolve all details of the $\zeta$-reduction, as discussed later in more detail. Nevertheless, we believe that our analysis is meaningful as a milestone for better understanding of the $\zeta$-reduction.

Before proceeding to the analysis of the modified FENE-PM model, we need to explain the symbols utilized in the analysis. The symbols in our analysis are chosen to be identical to those in our recent papers ${ }^{22,46}$ and are not necessarily identical to those utilized in the original paper ${ }^{34}$ for the FENE-PM model: For example, we prefer to use the symbols $\kappa, \sigma$, and $\tau$ for the FENE spring strength, the stress, and the relaxation time, respectively, whereas the original paper ${ }^{34}$ used these symbols for the velocity gradient tensor, the dyadic of end-to-end vector of the spring, and the stress. To avoid confusion, a reference table of those symbols is given in Appendix A.

\section{4-1. Constitutive Equation of Modified FENE-PM Model.}

Following Ref.34, we consider a system of unentangled bead-spring chains each being composed of $N$ beads and $N-1$ FENE springs. Each spring is composed of $g$ Kuhn segments, and the number of Kuhn segments per chain, $n_{\mathrm{K}}$, is related to $g$ and $N$ as $n_{\mathrm{K}}=(N-1) g$. Then, the stretchability parameter of the spring is given by

$$
b \equiv \frac{\kappa_{\mathrm{H}}\left\{g l_{\mathrm{K}}\right\}^{2}}{k_{\mathrm{B}} T}=3 g=\frac{3 n_{\mathrm{K}}}{N-1}
$$

where $k_{\mathrm{B}}$ and $T$ denote the Boltzmann constant and absolute temperature, $l_{\mathrm{K}}$ is the step length of the Kuhn segment, the factor $g l_{\mathrm{K}}$ represents the full-stretch length of the FENE spring, and $\kappa_{\mathrm{H}}=$ $3 k_{\mathrm{B}} T / g l_{\mathrm{K}}^{2}$ is the strength of a Hookean spring that has the mean-square end-to-end distance $g l_{\mathrm{K}}^{2}$ at equilibrium. In the FENE-PM model, ${ }^{34}$ all FENE springs are approximated to have the same strength $\kappa=f_{\mathrm{FENE}} \kappa_{\mathrm{H}}$ and the FENE factor $f_{\mathrm{FENE}}$, expressing the enhancement of the chain tension due to the FENE effect, is given by

$$
f_{\mathrm{FENE}}=\frac{1}{1-\left\langle\mathbf{u}^{2}\right\rangle /\left\{g l_{\mathrm{K}}\right\}^{2}}
$$

Here, $\left\langle\mathbf{u}^{2}\right\rangle$ is the mean-square end-to-end distance averaged for all FENE springs under 
flow/deformation. The rheological behavior in the steady flow state calculated with the above approximation is satisfactorily close to the behavior obtained from the more rigorous FENE-P model (because the conformation deduced from the FENE-P model is more or less similar for all springs)..$^{34}$

The FENE-PM model gives the constitutive equation that describes time evolution of the stress tensor $\boldsymbol{\sigma}_{j}$ associating to $j$-th eigenmode of orientation function of the chain $(j=1,2, . ., N-1)$. According to the definition of the stress given in Ref.34 for the case of uniaxial elongation in $z$ direction, the $z z$ components of this $\boldsymbol{\sigma}_{j}$ have negative values, and the $x x$ and $y y$ components, positive values. These signs of the stress components are just opposite to those used in the other definition, ${ }^{4-6}$ but the following calculation is consistent with the definition in Ref.34 to give correct results.

Under the elongation involving no rotational component of motion, the constitutive equation of $\boldsymbol{\sigma}_{j}$ is expressed in terms of just a few parameters, the strain rate $\dot{\varepsilon}, f_{\mathrm{FENE}}$ (eq 5), and the relaxation time $\tau_{j}^{[\mathrm{R}]}$ of $j$-th Rouse eigenmode being given by

$$
\tau_{j}^{[\mathrm{R}]}=\frac{\zeta}{2 \kappa_{\mathrm{H}} a_{j}}=\tau_{1}^{[\mathrm{R}]} \frac{\sin ^{2}(\pi / 2 N)}{\sin ^{2}(j \pi / 2 N)} \text { with } a_{j}=4 \sin ^{2}(j \pi / 2 N)
$$

In eq 6 , the factor $a_{j}$ denotes the Rouse eigenvalue of $j$-th mode. In the original FENE-PM model ${ }^{34}$ the friction coefficient $\zeta$ is a constant identical to $\zeta_{\text {eq }}$ at equilibrium. However, we can treat $\zeta$ as a variable that is the same for all segments (which is consistent with the pre-averaging approximation for the spring constant in the model) but changes its value with the chain stretch/orientation, or, with the stress. Adopting this treatment of $\xi$, we can re-formulate the constitutive equation (through a formal integration in the configurational space explained in Ref.34) for the normalized stress tensor, $\tilde{\boldsymbol{\sigma}}_{j}=\sigma_{j} / v k_{\mathrm{B}} T$ with $v$ being the number density of the chains. Specifically, for the elongation in $z$ direction, the constitutive equations for the $x x, y y$, and $z z$ components (diagonal components) of the stress tensor, $\tilde{\sigma}_{j, x x}, \tilde{\sigma}_{j, y y}$, and $\tilde{\sigma}_{j, z z}$, are written as

FENE-PM plus anisotropic $\zeta-$ reduction:

$$
\begin{aligned}
& \dot{\tilde{\sigma}}_{j, \xi \xi}=-\left\{\frac{X}{r_{\xi} \tau_{j}^{\mathrm{eq}}}+\dot{\varepsilon}-\frac{\dot{X}}{X}\right\} \tilde{\sigma}_{j, \xi \xi}+\dot{\varepsilon}-\frac{\dot{X}}{X} \quad(\xi=x, y) \\
& \dot{\tilde{\sigma}}_{j, z z}=-\left\{\frac{X}{r_{z} \tau_{j}^{\mathrm{eq}}}-2 \dot{\varepsilon}-\frac{\dot{X}}{X}\right\} \tilde{\sigma}_{j, z z}-2 \dot{\varepsilon}-\frac{\dot{X}}{X}
\end{aligned}
$$


with

$$
\begin{aligned}
& X(t, \dot{\varepsilon})=\frac{f_{\mathrm{FENE}}(t, \dot{\varepsilon})}{f_{\mathrm{FENE}}^{\mathrm{eq}}} \text { with } f_{\mathrm{FENE}}^{\mathrm{eq}}=\frac{b+3}{b} \\
& \tau_{j}^{\mathrm{eq}}=\frac{\zeta_{\mathrm{eq}}}{2 f_{\mathrm{FENE}}^{\mathrm{eq}} \kappa_{\mathrm{H}} a_{j}}=\frac{1}{f_{\mathrm{FENE}}^{\mathrm{eq}}} \tau_{j}^{[\mathrm{R}]} \\
& r_{\xi}(t, \dot{\varepsilon})=\frac{\zeta_{\xi}(t, \dot{\varepsilon})}{\zeta_{\mathrm{eq}}}(\leq 1) ; \quad \xi=x, y, z
\end{aligned}
$$

The factor $X$ (eq 9) is a normalized FENE factor defined on the basis of eq 5, and $\tau_{j}^{\text {eq }}$ (eq 10) is the equilibrium relaxation time of $j$-th eigenmode of the FENE-PM model that is directly related to the Rouse relaxation time $\tau_{j}^{[\mathrm{R}]}$ (eq 6). Finally, $r_{\xi}$ (eq 11) denotes the $\xi$-reduction ratio for the segmental motion in $\xi$ direction: Simulations by Hunt and $\operatorname{Todd}^{47}$ and by Ianniruberto and coworkers $^{21}$ suggest that the $\zeta$-reduction under elongational flow occurs anisotropically in $z$ and other two directions. Considering their simulation results, we have assumed the anisotropic $\zeta$-reduction in eqs 7,8 and 11. The $x$ and $y$ directions are equivalent under the uniaxial elongation, and we have considered this equivalence in the constitutive equation (eq 7).

Here, a comment needs to be made for this anisotropy in $\zeta$-reduction. Under the elongation, the segmental motion should occur differently in $z$ direction and other two directions (as also suggested from the simulation ${ }^{21,47}$ ) and thus we have considered this difference in eqs 7,8 , and 11 . Nevertheless, for our PS27 and PtBS53 melts, it turned out that the calculation based on eqs 7, 8, and 11 (explained later) give almost identical elongational behavior for the two cases of presence and absence of the anisotropy in the $\zeta$-reduction, as explained later in more detail. Thus, the magnitude of anisotropy is unknown for the PS27 and PtBS53 melts but this uncertainty introduces no ambiguity in our discussion presented later. At the same time, unfortunately, this lack of ambiguity means that the anisotropy, quite possibly emerging in our melts, cannot be resolved from comparison of the elongational data and the anisotropy-insensitive calculation.

The FENE factor defined by eq 5 can be related to the normalized stress components as ${ }^{34}$ $f_{\mathrm{FENE}}=(b+3) / b-\{b(N-1)\}^{-1} \sum_{j=1}^{N-1}\left\{2 \tilde{\sigma}_{j, x x}+\tilde{\sigma}_{j, z z}\right\} \quad$ (where a relationship $\tilde{\sigma}_{j, x x}=\tilde{\sigma}_{j, y y}$ reflecting the equivalence of $x$ and $y$ directions has been utilized). Consequently, eq 9 can be rewritten in terms of those stress components, the number of beads per chain $N$, and the stretchability parameter $b$ (eq 4 ):

$$
X=1-\frac{1}{(b+3)(N-1)} \sum_{j=1}^{N-1}\left(2 \tilde{\sigma}_{j, x x}+\tilde{\sigma}_{j, z z}\right)
$$


From eqs 7 and 8 combined with eq 12, we can calculate a consistent set of $X, \tilde{\sigma}_{j, z z}$, and $\tilde{\sigma}_{j, x x}\left(=\tilde{\sigma}_{j, y y}\right)$ according to the flow condition, i.e., in the steady state, or, on start-up/cessation of flow. Then, the normalized tensile stress, that can be directly compared with the experimental data, is obtained from $\tilde{\sigma}_{j, z z}$ and $\tilde{\sigma}_{j, x x}$ as $^{34}$

$$
\tilde{\sigma}_{\mathrm{E}} \equiv \frac{\sigma_{\mathrm{E}}}{v k_{\mathrm{B}} T}=\sum_{j=1}^{N-1}\left(\tilde{\sigma}_{j, x x}-\tilde{\sigma}_{j, z z}\right)
$$

The following two sections, sections 4-2 and 4-3, explain some details of this calculation. Readers who prefer to immediately see the message extracted from the calculation can skip those details and directly proceed to section 4-4.

\section{4-2. Steady State Elongational Viscosity.}

\section{4-2-1. formulation of normalized FENE factor and elongational viscosity.}

In the steady state, the time derivatives $\dot{\tilde{\sigma}}_{j, x x}, \dot{\tilde{\sigma}}_{j, z z}$, and $\dot{X}$ vanish and eqs 7 and 8 reduce to

$$
\begin{gathered}
\tilde{\sigma}_{j, x x}=\frac{r_{x} \frac{W i}{X}}{r_{x} \frac{W i}{X}+\frac{a_{j}}{a_{1}}} \quad(1 \leq j \leq N-1) \\
\tilde{\sigma}_{j, z z}=\frac{2 r_{z} \frac{W i}{X}}{2 r_{z} \frac{W i}{X}-\frac{a_{j}}{a_{1}}} \quad(1 \leq j \leq N-1)
\end{gathered}
$$

Here, $W i=\dot{\varepsilon} \tau_{1}^{\text {eq }}$ is the Weissenberg number defined in terms of the longest relaxation time $\tau_{1}^{\text {eq }}$ of the FENE-PM chain in the LVE regime. In the LVE regime (at equilibrium), the $\zeta$-reduction does not occur, i.e., $r_{\xi}=1$, but $\tau_{1}^{\mathrm{eq}}$ of the FENE-PM model differs from $\tau_{1}^{[\mathrm{R}]}$ of the Rouse model by the factor of $f_{\text {FENE }}^{\text {eq }}$ (cf. eq 10), because the Rouse model utilizes the Hookean spring, not the FENE spring. $W i$ defined as above (not $W i^{[\mathrm{R}]}=\dot{\varepsilon} \tau_{1}^{[\mathrm{R}]}$ defined for the Rouse model) corresponds to the Weissenberg number in experiments.

Substituting eqs 14 and 15 in eq 12, we find an equation that self-consistently determines the normalized FENE factor (elasticity enhancement factor) $X$, 


$$
X=1-\frac{2}{(b+3)(N-1)} W i\left(\sum_{j=1}^{N-1} \frac{r_{x}}{r_{x} W i+\frac{a_{j}}{a_{1}} X}+\sum_{j=1}^{N-1} \frac{r_{z}}{2 r_{z} W i-\frac{a_{j}}{a_{1}} X}\right)
$$

In eq $16, X$ is included in the left-hand side and also in the right-hand-side in two places (in the summation terms). Thus, for a given set of known parameters, $N, W i$, and $b$ (cf. eq 4), we can numerically solve eq 16 to determine $X$ given that we know the $\zeta$-reduction ratios, $r_{x}$ and $r_{z}$. Actually, for the given set of parameters, eq 16 has multiple solutions of $X$ (because rhs of eq 16 oscillates with an infinitely large amplitude on an increase of $W i$ ), as explained in Appendix B in more detail. We should choose the minimum value of the solutions of $X$ satisfying a physical requirement that $X$ never decreases with increasing $W i$ (namely, the chain is more stretched and stiffened under faster flow).

The $X$ value obtained as above is substituted in eqs 14 and 15 to give the values of $\tilde{\sigma}_{j, x x}$ and $\tilde{\sigma}_{j, z z}$. The elongational viscosity $\eta_{\mathrm{E}}(\dot{\varepsilon})$, normalized by $\eta_{\mathrm{E} 0}$ in the LVE regime, is calculated from these $\tilde{\sigma}_{j, \xi \xi}$ values as ${ }^{34}$

$$
\frac{\eta_{\mathrm{E}}(\dot{\varepsilon})}{\eta_{\mathrm{E} 0}}=\frac{F}{W i} \sum_{j=1}^{N-1}\left\{\tilde{\sigma}_{j, x x}-\tilde{\sigma}_{j, z z}\right\} \text { with } F=\frac{1}{2\left(N^{2}-1\right) \sin ^{2}(\pi / 2 N)}
$$

The factor $F$ is obtained from the calculation in the $\operatorname{LVE} \operatorname{limit}^{34}\left(\eta_{\mathrm{E}} / \eta_{\mathrm{E} 0} \rightarrow 1\right.$ for $\left.W i \rightarrow 0\right)$.

\section{4-2-2. assumption for $\zeta$-reduction ratio.}

Now we focus on the anisotropic $\xi$-reduction ratio $r_{\xi}$ that appears in eq 16 to determine $X$. Neither reliable experimental result nor a quantitative molecular theory is currently available for $r_{\xi}$. Thus, at this moment, we cannot compare, in a predictive sense, the model calculation explained above and the $\eta_{E}(\dot{\varepsilon}) / \eta_{E}(0)$ data of PS27k and PtBS53k melts shown in Figure 5. Considering this situation, we here test if the FENE-PM model modified for the anisotropic $\zeta$-reduction can mimic the data, given that the functional form of $r_{\xi}$ is adequately assumed. The $\xi$-reduction is expected to be stronger in $z$ direction (elongational direction) than in the other two directions, as also suggested from simulations..$^{21,47}$ Thus, we consider an extreme case specified as

Fully anisotropic $\zeta$-reduction: $r_{z}<1$ and $r_{x}=1$ (no reduction in $x$ direction)

For completeness, we also consider a hypothetical, opposite extreme case, 
The situation in our melt samples would/should be between these two extreme cases.

For both cases, the $\zeta$-reduction ratio $r_{z}$ (and $r_{x}$ for the isotropic reduction case) is believed to be determined by the chain orientation $S$ and the chain stretch ratio $\lambda$ and thus to be a function of the normalized tensile stress $\tilde{\sigma}_{\mathrm{E}}=\sigma_{\mathrm{E}} / v k_{\mathrm{B}} T\left(\sim S \lambda^{2}\right.$ with the decoupling approximation $){ }^{22,25,44}$ Nevertheless, for a given material in the steady flow state, $\tilde{\sigma}_{\mathrm{E}}$ can be regarded as a function of the Weissenberg number Wi. Thus, for comparison of the modified FENE-PM model with the steady state $\eta_{\mathrm{E}} / \eta_{\mathrm{E} 0}$ data, we may assume a supposedly reasonable form of $r_{z}(W i)$ as a function of Wi that describes monotonic decrease of $\zeta_{z}$ with Wi to examine if an adequate choice of parameters in this function allows the modified model to mimic the $\eta_{\mathrm{E}} / \eta_{\mathrm{E} 0}$ data. (This $r_{z}(W i)$ is later shown in Figure $8 \mathrm{a}$ as a function of $\tilde{\sigma}_{\mathrm{E}}$.)

We tested several functional forms of $r_{z}(W i)$ but could not find a simple analytic form being defined in the entire range of Wi and allowing the modified FENE-PM model to mimic the data. For this reason, we focused on $r_{z}(W i)$ defined separately in low- and high-Wi zones,

$$
r_{z}= \begin{cases}\exp \left(-A W i^{\alpha}\right) & \text { for } W i<W i_{\mathrm{c}} \\ B / W i^{\beta} & \text { for } W i>W i_{\mathrm{c}}\end{cases}
$$

$W i_{\mathrm{c}}$ is the $W i$ value at the boundary between the two zones, and the exponential form of $r_{z}$ for $W i<W i_{\mathrm{c}}$ guarantees $r_{z} \rightarrow 1$ for $W i \rightarrow 0$ as required from the definition of $r_{z}$ (eq 11). Because of the continuity of $r_{z}$ and its derivative $\mathrm{d} r_{z} / \mathrm{d} W i$ at the zone boundary, some parameters appearing in eq 19 are determined by the other parameters. Specifically, we chose $A, \alpha$, and $W i_{\mathrm{c}}$ as independent parameters that determine the remaining parameters:

$$
\beta=A \alpha W_{\mathrm{c}}^{\alpha}, \quad B=W_{\mathrm{c}}^{\beta} \exp \left(-A W_{\mathrm{c}}^{\alpha}\right)
$$

Table 2 summarizes the values of the independent parameters utilized in the following calculation. 
Table 2. Values of parameters for $r_{z}$ utilized in model calculation

\begin{tabular}{|l|c|c|c|c|}
\hline & & $A$ & $\alpha$ & $W i_{\text {c }}$ \\
\hline \multirow{3}{*}{ PS27 } & fully anisotropic case $^{\text {a }}$ (eq 18a) & 0.750 & 0.88 & 1.46 \\
\cline { 2 - 5 } & fully isotropic case $^{\mathrm{b}}$ (eq 18b) & 0.734 & 0.89 & 1.46 \\
\hline \multirow{2}{*}{ PtBS53 } & fully anisotropic case $^{\mathrm{a}}$ (eq 18a) & 0.760 & 1.21 & 0.86 \\
\cline { 2 - 5 } & fully isotropic case $^{\mathrm{b}}$ (eq 18b) & 0.750 & 1.21 & 0.86 \\
\hline
\end{tabular}

a: $r_{x}=1\left(\gg r_{z}\right.$ for $\left.W i \gg W i_{c}\right)$

b: $r_{x}=r_{z}$ (in the entire range of Wi)

\section{4-2-3. other parameters.}

The calculation with the modified FENE-PM model requires the model parameters shown in Table 2 as well as the following parameters related to the chain structure. The number of Kuhn segments per chain is experimentally known to be $n_{\mathrm{K}}=30$ and 35 for PS27k and PtBS53k, respectively (cf. Table 1). The number of the beads per chain, $N$, should be an integer by definition ( $N$ specifies the upper bound of summation over the mode index in eqs 16 and 17), and its value needs to be large enough to describe the relaxation mode distribution of the PS27k and PtBS53k chains occurring over several decades of time scale (cf. Figures 1 and 2 for the mode distribution in the LVE regime: This mode distribution in turn reflects the flexible character of these chains). Considering this point, we chose $N=16$ for both PS27k and PtBS53k, which automatically gave the stretchability parameter $b=3 n_{\mathrm{K}} /(N-1)=6$ and 7 (cf. eq 4) for PS27k and PtBS53k, respectively. In fact, moderate changes in our choice of $N$ does not significantly affect the $\eta_{\mathrm{E}} / \eta_{\mathrm{E} 0}$ ratio calculated from the FENE-PM model with no $\zeta$-reduction, as demonstrated in Appendix C. This insensitivity to the choice of $N$ was noted also for the model modified for $\zeta$-reduction. Namely, the characteristic feature of bead-spring models in general, the slow relaxation behavior of the model being not too much affected by the choice of $N$, is noted also for the FENE-PM model with/without $\zeta$-reduction. Of course, the model has a coarse-grained character to have a sound meaning only in a time scale longer the characteristic time $\tau_{N-1}$ of the fastest $(N-1$ th) relaxation mode considered in the model. The choice of $N=16$ explained above allows the model to have the sound meaning at $t>\tau_{15}^{\mathrm{eq}}=\tau_{1}^{\mathrm{eq}}\left\{\sin ^{2}(\pi / 2 N) / \sin ^{2}(j \pi / 2 N)\right\} \cong 0.4 \mathrm{~s}$ (cf. eqs 6 and $10 ; \tau_{1}^{\mathrm{eq}} \cong 40 \mathrm{~s}$ in the LVE regime), thereby covering the whole range of time of our interest for the stress evolution and relaxation of PS27 and PtBS53 melts. 


\section{4-2-4. glassy contribution.}

The above model calculation, based on eqs 14-20, does not include the glassy contribution to the elongational viscosity $\eta_{\mathrm{E}}$. This contribution is minor $(\sim 5 \%)$ in the LVE flow regime at low $\omega$ as explained for Figures 1 and 2, but not necessarily negligible at high $\omega$ and/or short $t$ (in both LVE and nonlinear regimes). Thus, we added this glassy contribution to the viscosity calculated from the modified FENE-PM model. Inoue and coworkers ${ }^{48,49}$ conducted rheo-optical analysis for PS melts to demonstrate that the glassy stress component exhibits significant strain-softening under elongation and the critical strain rate at the onset of this nonlinearity is $\dot{\varepsilon}_{\mathrm{c}, \mathrm{g}} \cong 10^{-3} / \tau_{\mathrm{g}}$, with $\tau_{\mathrm{g}}$ being the glassy relaxation time in the LVE regime. This strongly nonlinear character of the glassy relaxation was confirmed also from dielectric measurements under shear. ${ }^{50}$ Nevertheless, the strain rates examined for our PS27 and PtBS53 melts, $\dot{\varepsilon} / \mathrm{s}^{-1}=0.01-0.5$ (cf. Figures 3-7), were in the range of $\dot{\varepsilon} \leq 6 \times 10^{-4} / \tau_{\mathrm{g}}$ (with $\tau_{\mathrm{g}}$ being the glassy relaxation time summarized in eq 3 ) and thus below $\dot{\varepsilon}_{\mathrm{c}, \mathrm{g}}$. For this reason, we did not consider the nonlinearity of the glassy relaxation of those samples and evaluated the steady state elongational viscosity as $\eta_{\mathrm{E}}=\eta_{\mathrm{E}, \mathrm{FENE}-\zeta}+3 \eta_{0, \mathrm{~g}}$, where $\eta_{\mathrm{E}, \mathrm{FENE}-\zeta}$ is the viscosity calculated from the modified FENE-PM model (eqs 14-20) and $\eta_{0, \mathrm{~g}}$ is the contribution of the glassy relaxation to the zero-shear viscosity (evaluated from the green curves in Figures 1 and 2).

\section{4-2-5. comparison with data.}

For the extreme cases of fully anisotropic and isotropic $\zeta$-reduction specified by eqs $18 \mathrm{a}$ and $18 \mathrm{~b}$, the $\eta_{\mathrm{E}} / \eta_{\mathrm{E} 0}$ ratio of unentangled melt samples thus calculated from the modified FENE-PM model and the $\eta_{0, \mathrm{~g}}$ data are shown in Figure 5 with the small red circles and red curves, respectively. For comparison, the $\eta_{\mathrm{E}} / \eta_{\mathrm{E} 0}$ ratio calculated from the FENE-PM model with no $\zeta$-reduction $\left(r_{x}=r_{z}=1\right.$ in eqs 14-16) is shown with the black curves. Significant hardening due to the FENE effect (black curves) is largely suppressed by the $\zeta$-reduction incorporated in the modified model (red circle and red curve), and this model can excellently mimic the $\eta_{\mathrm{E}} / \eta_{\mathrm{E} 0}$ data in the entire range of Wi. In fact, the parameters summarized in Table 2 were chosen so as to achieve this quality of agreement between the modified FENE-PM calculation utilizing eq 19 and the data. (Of course, we should not assume too much molecular meaning for the functional form of the $\zeta$-reduction ratio specified by eq 19 and the values of the associating parameters $A, \alpha$, and 
$W i_{c}$ : The quality of agreement is determined only by the $r_{z}$ values (not its functional form) at respective Wi.) The excellent agreement between the model calculation and data in turn lends support to our empirical assignment explained earlier, the hardening at intermediate Wi due to the FENE effect weakened by the $\zeta$-reduction and the softening at high $W i$ due to the $\zeta$-reduction overwhelming the FENE effect.

In Figure 5, we also note that the model calculations for the extreme cases of fully anisotropic and isotropic $\zeta$-reduction (red circle and red curve) are indistinguishable and commonly fit the data very well. Namely, in the extreme cases, the $\zeta$-reduction ratio $r_{\mathrm{x}}$ is quite different but the ratio $r_{z}$ giving the excellent fit is almost identical (as noted from the close coincidence of the parameter values for those cases; cf. Table 2). This behavior of $r_{z}$ reflects the following feature of eq 16: In rhs of eq 16, $r_{\mathrm{x}}$ and $r_{\mathrm{z}}$ are included only in the first and second summations, respectively. For large $W i$, the second summation overwhelms the first summation and consequently the normalized FENE factor $X$ is mostly determined by $r_{z}$, so that the good fit in the two extreme cases is achieved with almost identical $r_{z}$, as explained in more detail in Appendix B. In fact, for general cases of anisotropic $\zeta$-reduction $\left(r_{z}<r_{x}<1\right)$, the same quality of fit was achieved with the same $r_{z}$ as utilized for the two extreme cases. Furthermore, the calculated results were insensitive to the anisotropy of $\zeta$-reduction also in the transient states after start-up/cessation of flow, as explained later. These results indicate that the lack of information for the anisotropy in actual PS27 and PtBS53 melts introduces no ambiguity in our discussion of the magnitude of $\zeta$-reduction presented below. (At the same time, unfortunately, this lack of ambiguity means that the anisotropy in those melts cannot be resolved from comparison of our model calculation and the data.)

In the modified FENE-PM calculation explained above, the $\zeta$-reduction ratio $r_{z}$ (and $r_{x}$ in the isotropic case; eq 18b) was treated as functions of Wi (eq 19). Nevertheless, for each value of Wi, the calculation gave the normalized tensile stress per chain, $\tilde{\sigma}_{\mathrm{E}}=\sigma_{\mathrm{E}} / v k_{\mathrm{B}} T$ (that was converted to the $\eta_{\mathrm{E}} / \eta_{\mathrm{E} 0}$ ratio in Figure 5), which allows us to plot $r_{z}$ against $\tilde{\sigma}_{\mathrm{E}}$ and re-define $r_{z}$ in the steady flow state as a function of $\tilde{\sigma}_{\mathrm{E}}$. This plot, shown in Figure 8a, quantifies the magnitude of $\xi$-reduction as a function of $\tilde{\sigma}_{\mathrm{E}}$ reflecting the chain conformation, and is utilized later in the transient state calculation as a reference converting $\tilde{\sigma}_{\mathrm{E}}$ into $r_{z}$.

For direct comparison between PS27 and PtBS53 having similar but not identical $n_{\mathrm{K}}$ values (30 and 35; cf. Table 1), Figure $8 \mathrm{~b}$ re-plots $r_{z}$ against the normalized stress per Kuhn segment, 
$\tilde{\tilde{\sigma}}_{\mathrm{E}}=\sigma_{\mathrm{E}} / n_{\mathrm{K}} v k_{\mathrm{B}} T\left(=\tilde{\sigma}_{\mathrm{E}} / n_{\mathrm{K}}\right)$. For $\tilde{\tilde{\sigma}}_{\mathrm{E}}<0.3$, the $\xi$-reduction occurs gradually for PS27 and PtBS53

to a very similar extent for both cases of anisotropic and isotropic $\zeta$-reduction shown with the small circles and curves, respectively. The calculation based on eqs 14-20 in turn indicated that the Wi values are smaller than $0.9_{4}$ for both PS27 and PtBS53 in the range of $\tilde{\tilde{\sigma}}_{\mathrm{E}}<0.3$. For $W i<0.9_{4}$, Figure 5 demonstrates no significant difference of the $\eta_{\mathrm{E}} / \eta_{\mathrm{E} 0}$ ratio for PS27 and PtBS53. In contrast, for $\tilde{\tilde{\sigma}}_{\mathrm{E}}>0.3$, the $\zeta$-reduction becomes considerably stronger for PS27 than for PtBS53 (by a factor of $\sim 5$ for $\tilde{\tilde{\sigma}}_{\mathrm{E}}=2$, for example), thereby giving smaller $\eta_{\mathrm{E}} / \eta_{\mathrm{E} 0}$ ratio for PS27 for $W i>$ 0.9 (Figure 5). This quantitative difference, being pursued through the model analysis, confirms the changes of the magnitude of $\zeta$-reduction with the chemical structure of the chain discussed for Figures 3-5.
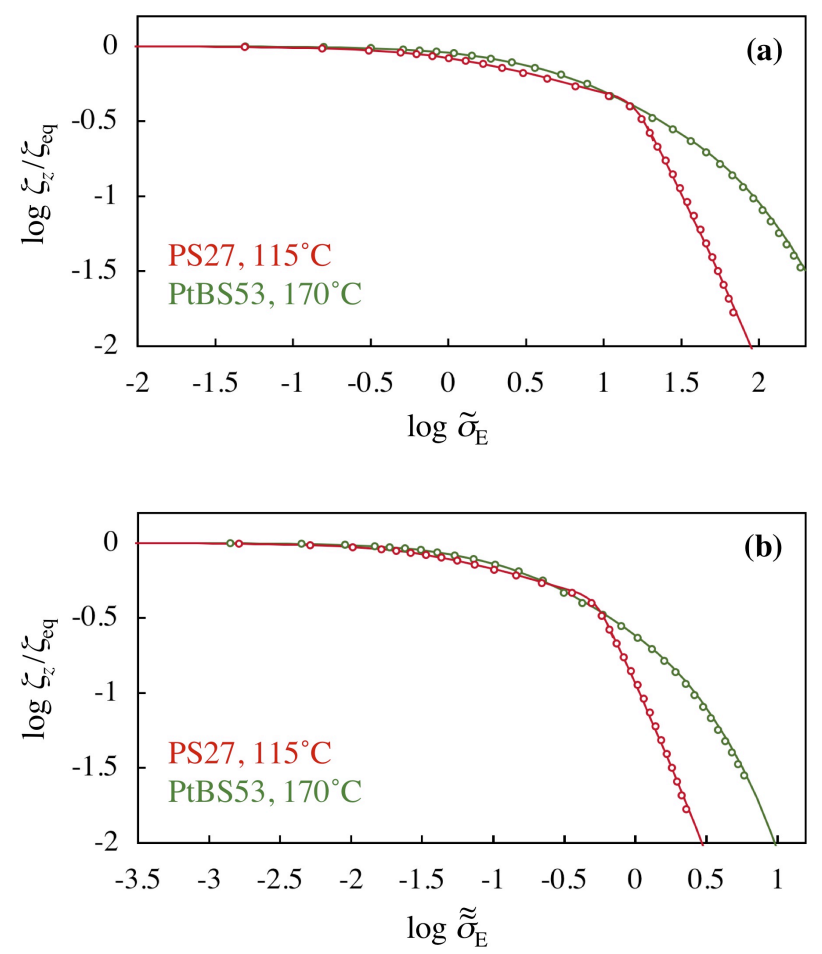

Figure 8. Friction reduction ratio $\zeta_{z} / \zeta_{\mathrm{eq}}\left(=r_{z}\right)$ utilized in the modified FENE-PM calculation in the steady flow state. Small circles indicate the ratio for the extreme case of anisotropic $\zeta$-reduction $\left(r_{z}<1, r_{x}=1\right)$, and the curves, the ratio for isotropic $\zeta$-reduction $\left(r_{z}=r_{x}<1\right)$. In panel (a), the $\zeta_{z} / \zeta_{\text {eq }}$ ratio is double-logarithmically plotted against the normalized tensile stress per chain, $\tilde{\sigma}_{\mathrm{E}}=\sigma_{\mathrm{E}} / v k_{\mathrm{B}} T$. In panel (b), the ratio is plotted against the normalized tensile stress per Kuhn segment, $\tilde{\tilde{\sigma}}_{\mathrm{E}}=\sigma_{\mathrm{E}} / n_{\mathrm{K}} v k_{\mathrm{B}} T$, for direct comparison between PS27 and PtBS53.

Here, a comment needs to be made for the isotropic $\zeta$-reduction ratio of entangled PS melts reported by Yaoita and coworkers ${ }^{22}$ and Ianniruberto and coworkers. ${ }^{21}$ Because their methods of evaluating this ratio are different from our method giving the plots in Figure 8, direct comparison between their and our results might introduce some artifact. Nevertheless, it is still informative to 
compare the results for entangled and unentangled PS melts. This comparison is presented in Appendix D. It turned out that the isotropic $\zeta$-reduction ratio reported by Yaoita and coworkers ${ }^{22}$ and by Ianniruberto and coworkers ${ }^{21}$ was moderately larger ( $\xi$-reduction was weaker), by a factor of 2-3, compared to the ratio $r_{z}$ shown in Figure 8. This result might indicate that the isotropic $\zeta$-reduction ratio evaluated for entangled PS melts was a sort of average between the ratios $r_{x}$ and $r_{z}$ in $x$ and $z$ directions in the realistic case of anisotropic reduction. However, we cannot rule out, at this moment, a possibility that the ratio reported for entangled PS was not of purely frictional nature due to the methods of evaluating the ratio, as explained in Appendix D in more detail. The other possibility is related to distributions of the spring stiffness and the friction coefficient among the segments of a chain. In the modified FENE-PM model, these distribution are pre-averaged, and the average might weigh more heavily on less frictional segments connected to more stretched springs than treated in literature ${ }^{21,22}$ (cf. Appendix D). These possibilities deserve further attention.

\section{4-3. Calculation of Stress Growth and Relaxation on Start-up and Cessation of Flow.}

The FENE-PM model modified for the $\zeta$-reduction can satisfactorily mimic the steady state $\eta_{\mathrm{E}}$ data of the unentangled PS27 and PtBS53 samples given that the parameters (cf. Table 2) are adequately chosen, as explained for Figure 5. Nevertheless, this result does not mean that the feature of $\zeta$-reduction has been fully elucidated, because no transient change of $\zeta$ occurs in the steady flow state. From this point of view, it is important to examine the behavior of the modified FENE-PM model for the stress growth and relaxation on start-up and cessation of flow.

For this purpose, we firstly need to specify how the $\zeta$-reduction ratios $r_{x}$ and $r_{z}$ (eq 11) change with $t$ in the transient state. In general, the segmental friction $\zeta$ is believed to be determined by the orientational anisotropy $S$ and the stretch ratio $\lambda$ of the chain and behave as a function of the normalized tensile stress $\tilde{\sigma}_{\mathrm{E}}$ (that scales as $S \lambda^{2}$ within the decoupling approximation). ${ }^{22,25,42}$ Thus, in the simplest treatment, we can utilize the plots of $r_{x}$ and $r_{z}$ shown in Figure 8a to convert the time-dependent $\tilde{\sigma}_{\mathrm{E}}$ value in the transient state into the time-dependent $r_{x}$ and $r_{z}$ values, i.e., $r_{x}=$ $r_{x}\left\{\tilde{\sigma}_{\mathrm{E}}(t)\right\}$ and $r_{z}=r_{z}\left\{\tilde{\sigma}_{\mathrm{E}}(t)\right\}$. The time-dependent changes of $\tilde{\sigma}_{\mathrm{E}}(t)$ are in turn calculated from eqs 7 and 8 that include $r_{x}$ and $r_{y}$. Namely, in this treatment, eqs 7 and 8 combined with this “converter" (Figure 8a) are solved to give a consistent set of $\tilde{\sigma}_{\mathrm{E}}(t), r_{x}\left\{\tilde{\sigma}_{\mathrm{E}}(t)\right\}$, and $r_{z}\left\{\tilde{\sigma}_{\mathrm{E}}(t)\right\}$ on start-up and cessation of flow. Details of this consistent calculation are explained in Supporting 
Information, and a brief summary is given below.

\section{4-3-1. stress growth on start-up of flow.}

We first focus on start-up of elongational flow. For this case, eqs 7, 8, 12, and 13 hold without any modification, and the initial conditions for the variables therein (just before the start-up of flow) are specified as $\tilde{\sigma}_{j, x x}(0)=\tilde{\sigma}_{j, z z}(0)=0, r_{x}(0)=r_{z}(0)=1$, and $X(0)=1$. As explained in Section A of Supporting Information, eqs 7, 8, and 12 allow us to express the increments of the normalized FENE factor, $\Delta X$, and of the normalized stress components, $\Delta \tilde{\sigma}_{j, \xi \xi}$, in a very short interval of time from $t$ to $t+\Delta t$ (with $\Delta t<<\tau_{N-1}^{\mathrm{eq}}$ ) in terms of $X, \tilde{\sigma}_{j, \xi \xi \xi}$, and the normalized tensile stress $\tilde{\sigma}_{\mathrm{E}}$ at time $t$. The results are summarized below.

$$
\Delta X=\frac{X}{A}(1-X-B)
$$

with

$$
\begin{aligned}
A= & X+\frac{2\left\{(\dot{\varepsilon} \Delta t-1) S_{x 1}+S_{x 2}\right\}}{(b+3)(N-1)} \exp \{-\dot{\varepsilon} \Delta t\} \\
& -\frac{\left\{(2 \dot{\varepsilon} \Delta t+1) S_{z 1}-S_{z 2}\right\}}{(b+3)(N-1)} \exp \{2 \dot{\varepsilon} \Delta t\} \\
B= & \frac{2\left\{\dot{\varepsilon} \Delta t S_{x 1}+S_{x 2}\right\}}{(b+3)(N-1)} \exp \{-\dot{\varepsilon} \Delta t\} \\
& -\frac{\left\{2 \dot{\varepsilon} \Delta t S_{z 1}-S_{z 2}\right\}}{(b+3)(N-1)} \exp \{2 \dot{\varepsilon} \Delta t\} \\
S_{\xi 1}= & \sum_{j=1}^{N-1} \exp \left\{-\frac{1}{\tau_{j}^{\mathrm{eq}}} \frac{X}{r_{\xi}\left\{\tilde{\sigma}_{\mathrm{E}}\right\}} \Delta t\right\} \quad(\xi=x, z) \\
S_{\xi 2}= & \sum_{j=1}^{N-1} \tilde{\sigma}_{j, \xi \xi} \exp \left\{-\frac{1}{\tau_{j}^{\mathrm{eq}}} \frac{X}{r_{\xi}\left\{\tilde{\sigma}_{\mathrm{E}}\right\}} \Delta t\right\} \quad(\xi=x, z)
\end{aligned}
$$

and

$$
\begin{aligned}
\Delta \tilde{\sigma}_{j, x x}=-\tilde{\sigma}_{j, x x}+ & \left\{\frac{\Delta X}{X}\left(\tilde{\sigma}_{j, x x}+\dot{\varepsilon} \Delta t-1\right)+\left(\tilde{\sigma}_{j, x x}+\dot{\varepsilon} \Delta t\right)\right\} \times \\
& \exp \left\{-\frac{1}{\tau_{j}^{\mathrm{eq}}} \frac{X}{r_{x}\left\{\tilde{\sigma}_{\mathrm{E}}\right\}} \Delta t\right\} \exp \{-\dot{\varepsilon} \Delta t\}
\end{aligned}
$$




$$
\begin{aligned}
\Delta \tilde{\sigma}_{j, z z}=-\tilde{\sigma}_{j, z z}+ & \left\{\frac{\Delta X}{X}\left(\tilde{\sigma}_{j, z z}-2 \dot{\varepsilon} \Delta t-1\right)+\left(\tilde{\sigma}_{j, z z}-2 \dot{\varepsilon} \Delta t\right)\right\} \times \\
& \exp \left\{-\frac{1}{\tau_{j}^{\mathrm{eq}}} \frac{X}{r_{z}\left\{\tilde{\sigma}_{\mathrm{E}}\right\}} \Delta t\right\} \exp \{2 \dot{\varepsilon} \Delta t\}
\end{aligned}
$$

The $\zeta$-reduction ratios appearing in eqs 24-27, $r_{x}\left\{\tilde{\sigma}_{\mathrm{E}}\right\}$ and $r_{z}\left\{\tilde{\sigma}_{\mathrm{E}}\right\}$ at time $t$, are determined from $\tilde{\sigma}_{\mathrm{E}}$ at that time with the aid of the "converter" (Figure 8a).

The normalized tensile stress increment in the interval $\Delta t, \Delta \tilde{\sigma}_{\mathrm{E}}=\sum_{j=1}^{N-1}\left\{\Delta \tilde{\sigma}_{j, x x}-\Delta \tilde{\sigma}_{j, z z}\right\}$ (cf. eq 13), is evaluated from $\Delta \tilde{\sigma}_{j, x x}$ and $\Delta \tilde{\sigma}_{j, z z}$ given by eqs 26 and 27. Accumulating this $\Delta \tilde{\sigma}_{\mathrm{E}}$ from time 0 to $t$, we evaluated a contribution of the rubbery relaxation to $\tilde{\sigma}_{\mathrm{E}}$ at time $t$ (and calculated $r_{x}\left\{\tilde{\sigma}_{\mathrm{E}}\right\}$ and $r_{z}\left\{\tilde{\sigma}_{\mathrm{E}}\right\}$ from this $\tilde{\sigma}_{\mathrm{E}}$, as explained above). The glassy contribution was evaluated from the $G_{\mathrm{g}}$ * data in the LVE regime, because the strain rates $\dot{\varepsilon}$ examined in our experiments were below the critical rate $\dot{\varepsilon}_{\mathrm{c}, \mathrm{g}}\left(\cong 10^{-3} / \tau_{\mathrm{g}}\right)$ at the onset of glassy nonlinearity, as explained in the previous section. Dividing a sum of these contributions by the strain rate $\dot{\varepsilon}$, we finally obtained the elongational stress growth coefficient, $\eta_{\mathrm{E}}^{+}(t, \dot{\varepsilon})=v k_{\mathrm{B}} T \tilde{\sigma}_{\mathrm{E}} / \dot{\varepsilon}$, deduced from the modified FENE-PM model on the basis of the simplest treatment of transient changes of the $\zeta$-reduction ratio $r_{\xi}$ (assuming that the ratio at a given time is fully determined by the stress value at that time).

For the PS27 and PtBS53 samples, $\eta_{\mathrm{E}}^{+}(t, \dot{\varepsilon})$ thus calculated has been shown in Figures 3 and 4 with the colored asterisk $(*)$ and thick solid curve for the two extreme cases of fully anisotropic and isotropic $\zeta$-reduction. The calculated results are almost indistinguishable for these cases, because the normalized FENE factor $X$ is mostly determined by the second summation in eq 16 that involves only $r_{z}$, as explained in more detail in Appendix C. This result indicates that the uncertainty of the anisotropy in the $\zeta$-reduction in PS27 and PtBS53 melts does not disturb our discussion of $\zeta$-reduction, as explained earlier for Figure 5. This discussion, made for the transient $\eta_{\mathrm{E}}^{+}(t, \dot{\varepsilon})$ data, is presented later together with that for the stress relaxation.

Here, a comment needs to be made for the modified FENE-PM calculation in the LVE regime. In Figures 3 and 4, black solid and dashed curves indicate this LVE behavior with and without the contribution from the glassy relaxation. In the range of $t>1 \mathrm{~s}$ examined therein, this glassy contribution has fully grown to its steady state to have a value of $\sim 0.05 \eta_{\mathrm{E} 0}$, with $\eta_{\mathrm{E} 0}$ being the steady state elongational viscosity of the sample in the LVE regime. This glassy contribution is minor for the steady state $\eta_{\mathrm{E}}$ but non-negligibly contributes to the transient $\eta_{\mathrm{E}}^{+}(t)$ at short times 
(where the rubbery contribution has not largely grown), as clearly noted from the difference between the black solid and dashed curves in Figures 3 and 4 at $t<10 \mathrm{~s}$. Considering this point, we have added the glassy contribution to all modified FENE-PM calculations presented in this article.

\section{4-3-2. stress relaxation after cessation of flow.}

Now, we turn our attention to stress relaxation after cessation of flow imposed over a period of time $t^{*}$ (that corresponds to the Hencky strain $\varepsilon=2.5$ in our experiments for PS27 and PtBS53). For this relaxation process, eqs 12 and 13 hold as they are, but eqs 7 and 8 are modified by setting $\dot{\varepsilon}$ therein to be zero. The initial values of the variables therein (just before the flow cessation) are specified by

$$
\begin{aligned}
& \tilde{\sigma}_{\mathrm{E}}(0)=\tilde{\sigma}_{\mathrm{E}}^{\text {start -up }}\left(t^{*}\right), \quad \tilde{\sigma}_{j, \xi \xi}(0)=\tilde{\sigma}_{j, \xi \xi}^{\text {start -up }}\left(t^{*}\right) \\
& r_{\xi}\left\{\tilde{\sigma}_{\mathrm{E}}(0)\right\}=r_{\xi}\left\{\tilde{\sigma}_{\mathrm{E}}^{\text {start-up }}\left(t^{*}\right)\right\}, \quad X(0)=X^{\text {start-up }}\left(t^{*}\right)
\end{aligned}
$$

Here, the superscript "start-up" stands for the variables for the start-up of flow (with the flow time $\left.t^{*}\right)$. Those initial values are calculated by accumulating $\Delta X$ and $\Delta \tilde{\sigma}_{j, \xi \xi}$ specified by eqs 21-27.

As explained in Section B of Supporting Information, eqs 7 and 8 (with $\dot{\varepsilon}=0$ ) being combined with eq 12 give the increment of the normalized FENE factor, $\Delta X$, in a very short interval of time from $t$ to $t+\Delta t\left(\Delta t<<\tau_{N-1}^{\mathrm{eq}}\right)$ :

$$
\Delta X=\frac{1}{A^{\prime}}\left(1-X-B^{\prime}\right)
$$

with

$$
\begin{aligned}
A^{\prime}= & 1+\frac{B^{\prime}}{X}-\frac{1}{X(b+3)(N-1)} \times \\
B^{\prime}= & \left.\frac{1}{(b+3)(N-1)} \times 2 \exp \left\{-\frac{1}{\tau_{j}^{\mathrm{eq}}} \frac{X}{r_{x}\left\{\tilde{\sigma}_{\mathrm{E}}\right\}} \Delta t\right\}+\exp \left\{-\frac{1}{\tau_{j}^{\mathrm{eq}}} \frac{X}{r_{z}\left\{\tilde{\sigma}_{\mathrm{E}}\right\}} \Delta t\right\}\right) \\
& \sum_{j=1}^{N-1}\left(2 \sigma_{j, x x} \exp \left\{-\frac{1}{\tau_{j}^{\mathrm{eq}}} \frac{X}{r_{x}\left\{\tilde{\sigma}_{\mathrm{E}}\right\}} \Delta t\right\}+\sigma_{j, z z} \exp \left\{-\frac{1}{\tau_{j}^{\mathrm{eq}}} \frac{X}{r_{z}\left\{\tilde{\sigma}_{\mathrm{E}}\right\}} \Delta t\right\}\right)
\end{aligned}
$$

The corresponding expression of the increments of the normalized stress components, $\Delta \tilde{\sigma}_{j, \xi \xi \xi}$, is given by (cf. Section B of Supporting Information) 


$$
\begin{aligned}
\Delta \sigma_{j, \xi \xi}=-\sigma_{j, \xi \xi}+ & \left\{\sigma_{j, \xi \xi}+\frac{\Delta X}{X}\left(\sigma_{j, \xi \xi}-1\right)\right\} \times \\
& \exp \left\{-\frac{1}{\tau_{j}^{\mathrm{eq}}} \frac{X}{r_{\xi}\left\{\tilde{\sigma}_{\mathrm{E}}\right\}} \Delta t\right\} \quad(\xi=x, z)
\end{aligned}
$$

Following the simplest treatment explained earlier, we determined the $\zeta$-reduction ratios appearing in eqs 30-32, $r_{x}\left\{\tilde{\sigma}_{\mathrm{E}}\right\}$ and $r_{z}\left\{\tilde{\sigma}_{\mathrm{E}}\right\}$ at time $t$, from $\tilde{\sigma}_{\mathrm{E}}$ at that time with the aid of the "converter" (Figure 8a).

The increment of the normalized tensile stress in the interval $\Delta t, \Delta \tilde{\sigma}_{\mathrm{E}}=\sum_{j=1}^{N-1}\left\{\Delta \tilde{\sigma}_{j, x x}-\Delta \tilde{\sigma}_{j, z z}\right\}$, is obtained from $\Delta \tilde{\sigma}_{j, x x}$ and $\Delta \tilde{\sigma}_{j, z z}$ given by eq 32. Accumulating this $\Delta \tilde{\sigma}_{\mathrm{E}}$ from time 0 to $t$, we evaluated a contribution of the rubbery relaxation to $\tilde{\sigma}_{\mathrm{E}}$ (that determined $r_{x}\left\{\tilde{\sigma}_{\mathrm{E}}\right\}$ and $r_{z}\left\{\tilde{\sigma}_{\mathrm{E}}\right\}$, as explained above). The glassy contribution was evaluated from the $G_{\mathrm{g}}{ }^{*}$ data in the LVE regime (because the flow in our experiments was slow enough for the glassy relaxation, as explained in the previous sections). Adding these contributions, we finally obtained the relaxing stress $\tilde{\sigma}_{\mathrm{E}}^{-}(t)$ deduced from the modified FENE-PM model on the basis of the simplest treatment of the transient change of the $\zeta$-reduction ratio. (The superscript "-" explicitly shows that $\tilde{\sigma}_{\mathrm{E}}^{-}(t)$ is the stress relaxing after the flow cessation.) This $\tilde{\sigma}_{\mathrm{E}}^{-}(t)$, being reduced by $\tilde{\sigma}_{\mathrm{E}}^{-}\left(t_{\mathrm{r}}\right)$ at the reference time $t_{\mathrm{r}}=0.4 \mathrm{~s}$, has been shown in Figures 6 and 7 with the colored asterisk (*) and thick solid curve for the two extreme cases of fully anisotropic and isotropic $\zeta$-reduction. The calculated result is almost indistinguishable for these cases because $X$ determined by eq 16 is rather insensitive to $r_{x}$, as briefly mentioned for Figures 3-5 and explained in more detail in Appendix C. Thus, the uncertainty of the anisotropy in the $\zeta$-reduction in PS27 and PtBS53 melts introduces no ambiguity in our discussion of the transient changes of the $\zeta$-reduction presented in the following section.

Here, it is informative to compare the contributions of the FENE effect and $\zeta$-reduction to the acceleration of relaxation immediately after cessation of flow. For this purpose, we focus on the rubbery component of the tensile stress $\tilde{\sigma}_{\mathrm{E}-\mathrm{r}}^{-}(t)$ (without the glassy contribution) calculated from the modified FENE-PM model. Both FENE effect and $\xi$-reduction accelerate the relaxation of this $\tilde{\sigma}_{\mathrm{E}-\mathrm{r}}^{-}(t)$. As a reference, we calculate $\tilde{\sigma}_{\mathrm{E}-\mathrm{r}}^{-}(t)$ from the original FENE-PM model without $\xi$-reduction but for the initial condition (eq 28) identical to that for the modified model. This reference specifies the acceleration due only to the FENE effect in the modified model. Comparison of the relaxation behavior of the modified and original models is presented in 
Appendix E. It turned out that the initial relaxation after cessation of fast flow is much faster in the modified model than in the original model and thus the acceleration due to $\zeta$-reduction is much more significant than that due to the FENE effect. For example, for the highest $\dot{\varepsilon}\left(=0.5 \mathrm{~s}^{-1}\right)$ examined, the acceleration due to the $\zeta$-reduction is stronger than the acceleration due to the FENE effect by a factor of $\sim 30$ and 20 for PS27 and PtBS53, respectively; see Appendix E for further details. The acceleration in actual PS27 and PtBS53 melts on cessation of fast flow would have been similarly dominated by the $\zeta$-reduction.

\section{4-4. Transient Changes of $\zeta$-Reduction Ratio.}

As noted in Figures 3 and 4, the elongational stress growth coefficient $\eta_{\mathrm{E}}^{+}(t, \dot{\varepsilon})$ calculated from the modified FENE-PM model (colored asterisks and thick curves for the cases of fully anisotropic and isotropic $\zeta$-reduction) agrees with the data (circles) almost within 20\%, and the calculation works reasonably in this sense. In particular, for the highest $\dot{\varepsilon}$ examined $\left(=0.5 \mathrm{~s}^{-1}\right)$, the calculation without the $\zeta$-reduction (thin red curve) gives too fast growth compared to the data (red circles), and the $\zeta$-reduction significantly retards the growth (thick red curve) to reduce the difference between the calculation and data. From these results together with the close agreement between the model calculation and the steady state $\eta_{\mathrm{E}}(\dot{\varepsilon})$ data (Figure 5), one might consider that the modified FENE-PM model works well for the unentangled PS27 and PtBS53 melts. However, this is not the case. The stress growth for large Wi calculated for PS27 is considerably slower than observed; see the thick red curve calculated for $\dot{\varepsilon}=0.5 \mathrm{~s}^{-1}$ in Figure 3. The corresponding difference between the modified FENE-PM calculation and the data is more clearly noted for the stress relaxation after cessation of flow, as explained below in more detail.

In Figures 6 and 7, we note that the calculated terminal relaxation rate, seen as the slope of the curves at long $t \geq \tau_{1}^{\mathrm{eq}}(\sim 40 \mathrm{~s}$; cf. eq 2$)$, agrees well with the data. In fact, for small $\dot{\varepsilon}$, the agreement between the calculation and data is noted in the entire range of $t$ (because the nonlinearities due to the FENE effect and $\zeta$-reduction are minor for small $\dot{\varepsilon}$ ). However, for large $\dot{\varepsilon}$, the $\tilde{\sigma}_{\mathrm{E}}^{-}(t) / \tilde{\sigma}_{\mathrm{E}}^{-}\left(t_{\mathrm{r}}\right)$ ratio at long $t$ is considerably larger for the calculation, for example, by a factor of $\sim 2(200 \%)$ for $\dot{\varepsilon}=0.5 \mathrm{~s}^{-1}$ in Figure 6 . This difference is mostly attributed to a difference in the fast relaxation behavior shown in Figures 9 and 10 (that magnify the plots and curves in Figures 6 and 7 in the range of $t<5 \mathrm{~s}$ ). 


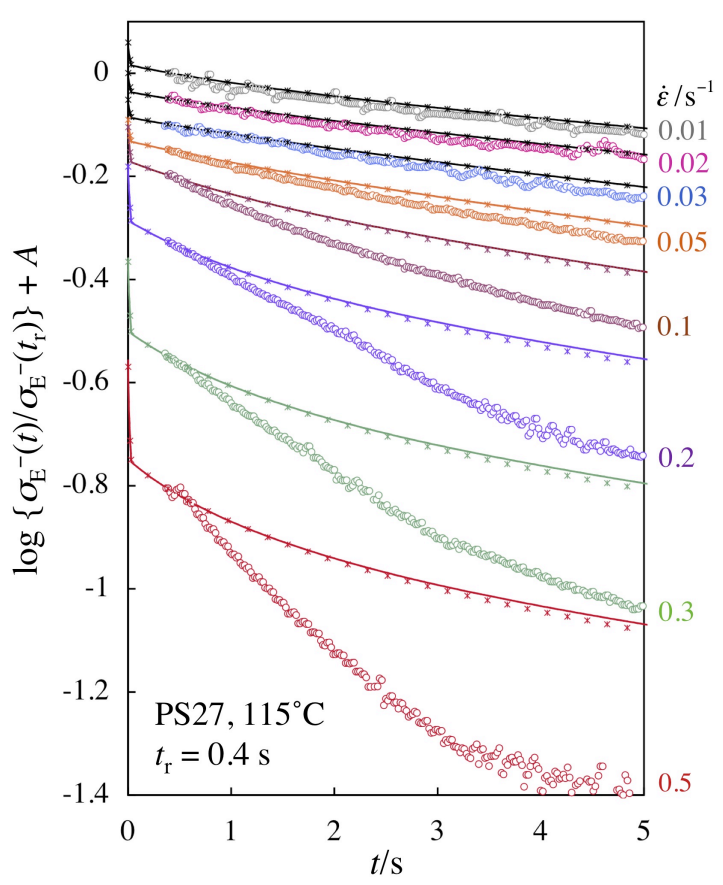

Figure 9. Magnified plot of the $\sigma_{\mathrm{E}}^{-}(t) / \sigma_{\mathrm{E}}^{-}\left(t_{\mathrm{r}}\right)$ ratio for PS27 at short $t(<5 \mathrm{~s})$. (The plot covering the entire range of $t$ is shown in Figure 6.) Asterisks (*) and curves indicate the $\sigma_{\mathrm{E}}^{-}(t) / \sigma_{\mathrm{E}}^{-}\left(t_{\mathrm{r}}\right)$ ratio calculated from the FENE-PM model modified for anisotropic and isotropic $\zeta$-reduction, respectively. The color of the asterisk and curve is the same as that of data (circle), except for the black asterisks and curves that are overlapping with the data (and not clearly visible if they have the same color as the data). For clarity of the plot, data and calculation for different strain rates are shifted vertically by adequate amount $A$.

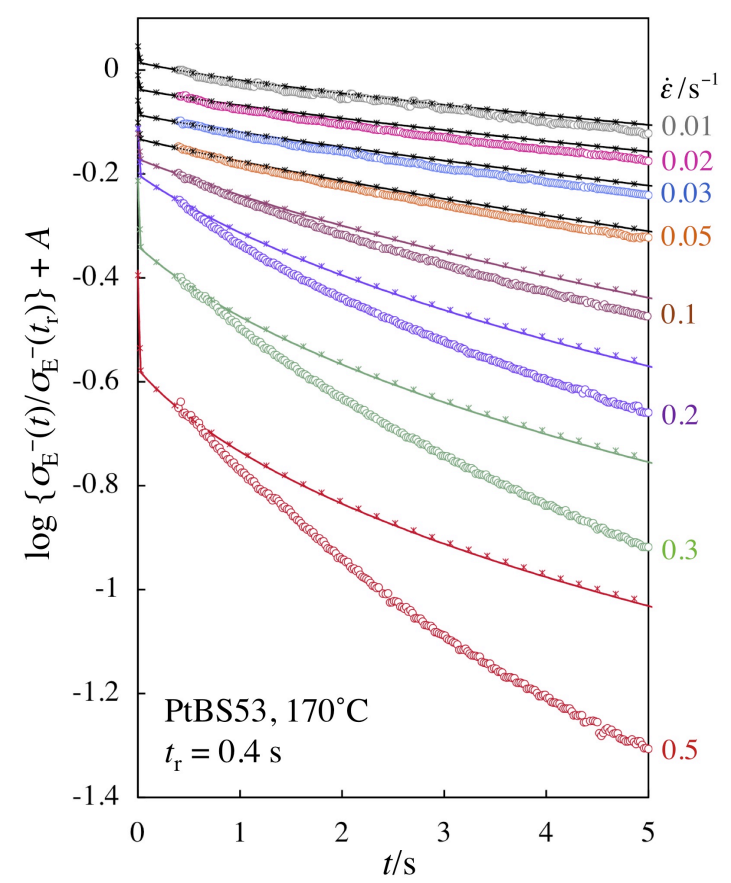

Figure 10. Magnified plot of the $\sigma_{\mathrm{E}}^{-}(t) / \sigma_{\mathrm{E}}^{-}\left(t_{\mathrm{r}}\right)$ ratio for PtBS53 at short $t(<5 \mathrm{~s})$. (The plot covering the entire range of $t$ is shown in Figure 7.) Asterisks $(*)$ and curves indicate the $\sigma_{\mathrm{E}}^{-}(t) / \sigma_{\mathrm{E}}^{-}\left(t_{\mathrm{r}}\right)$ ratio calculated from the FENE-PM model modified for anisotropic and isotropic $\zeta$-reduction, respectively. The color of the asterisk and curve is the same as that of data (circle), except for the black asterisks and curves that are overlapping with the data (and not clearly visible if they have the same color as the data). For clarity of the plot, data and calculation for different strain rates are shifted vertically by adequate amount $A$. 
As noted in Figures 9 and 10, the model calculation agrees well with the data at very short $t(<1$ s). Nevertheless, for large $\dot{\varepsilon}$, the calculated relaxation slows down at intermediate $t$ (up to $5 \mathrm{~s}$ ) whereas this slowing down is much less significant for the data, which results in a considerable difference in the magnitude of the $\tilde{\sigma}_{\mathrm{E}}^{-}(t) / \tilde{\sigma}_{\mathrm{E}}^{-}\left(t_{\mathrm{r}}\right)$ ratio emerging already at $t=5 \mathrm{~s}$. For example, for $\dot{\varepsilon}=0.5 \mathrm{~s}^{-1}$ in Figure 9, the magnitude difference by a factor of $\sim 2(200 \%)$ is noted at $t=5 \mathrm{~s}$, and this difference survives up to the terminal relaxation zone at $t>\tau_{1}^{\mathrm{eq}}$ (cf. Figure 6).

The above results strongly suggest failure of the simplest treatment adopted in the calculation that assumes $r_{\xi}$ at a time $t$ to be fully determined by $\tilde{\sigma}_{\mathrm{E}}$ at that $t$. Namely, in actual PS27 and PtBS53 melts, the friction $\zeta$ of a given segment does not seem to immediately adjust itself to the transient change in the oriented/stretched environment: This environment is an average contributed from many surrounding segments, and its transient change is unequivocally reflected in the change of $\tilde{\sigma}_{\mathrm{E}}$ with $t$. The given segment adjusts itself to this transient change of the environment, but this adjustment requires motion of that segment for exploring all configurations allowed by the change in the environment, and would occur with a delay. Because of this delay, the change of $\zeta$ of the given segment could be slower than the change of $\tilde{\sigma}_{\mathrm{E}}\left(\sim S \lambda^{2}\right.$, with $S$ and $\lambda$ being the orientational anisotropy and stretch ratio averaged for many surrounding segments), thereby leading to the failure of the simplest treatment considering no delay.

This argument is consistent with the observed stress growth and relaxation behavior: On start-up of flow, $\zeta$ decreases from $\zeta_{\mathrm{eq}}$ at equilibrium in both experiment and modified FENE-PM calculation. However, in the calculation adopting the simplest treatment of $\zeta$, this decrease occurs more quickly compared to the experiment, which would have led to the slower growth of the calculated $\eta_{\mathrm{E}}^{+}(t, \dot{\varepsilon})$ at large $\dot{\varepsilon}$ compared to the experiment; cf. Figures 3 and 4 . (The faster $\zeta$-reduction in the modified FENE-PM calculation tends to stretch the chain less efficiently in the transient state to result in this slower growth.) Correspondingly, after the flow cessation, $\zeta$ increases from $\zeta_{\text {flow }}$ under flow toward $\zeta_{\text {eq }}$ but this increase is faster in the modified FENE-PM calculation than in the experiment, which led to the slower relaxation of the calculated $\tilde{\sigma}_{\mathrm{E}}^{-}(t) / \tilde{\sigma}_{\mathrm{E}}^{-}\left(t_{\mathrm{r}}\right)$ ratio at intermediate $t$ for large $\dot{\varepsilon}$; cf. Figures 9 and 10. (At sufficiently long $t$ where $\zeta$ coincides with $\zeta_{\text {eq }}$ in both calculation and experiment, the calculated relaxation rate agrees with the observed terminal relaxation rate, $1 / \tau_{1}^{\mathrm{eq}} ; \mathrm{cf}$. Figures 6 and 7)

This argument suggests that the description of the $\zeta$-reduction and of the corresponding 
nonlinear stress growth/relaxation can be improved if the delay in the change of $\zeta$ (reflecting the segment motion required for adjustment of $\zeta$ ) is properly accounted in the molecular model(s) and/or simulation. This improvement, possibly achieved through a partially non-mean-field treatment of the segment friction in the model/simulation, is considered as an important subject of future work.

At the same time, we also note that the above argument is totally based on the starting model in our analysis, the FENE-PM model with the pre-averaging approximation for the spring stiffness. If this approximation severely fails in the actual PS27 and PtBS53 melts, we need to consider a distribution of the spring stiffness in the chain according to the stretch of respective springs. This distribution leads to a distribution of the local stress of the springs and consequently to a distribution of the friction coefficient $\xi$ along the chain backbone. These distributions might allow less stretched (less stiffened) springs and more frictional segments to dominate the transient rheological responses to reduce the difference between the calculation and the data in the transient state. This possibility is to be also examined in future work together with the possibility of the delay in the change of $\zeta$ explained above.

\section{CONCLUDING REMARKS}

In this study, we have examined nonlinear elongational rheology of unentangled PS27 and PtBS53 melts having nearly the same number of Kuhn segments per chain, $n_{\mathrm{K}}=30$ and 35 . For both PS27 and PtBS53, the steady state elongational viscosity $\eta_{\mathrm{E}}$ exhibited the strain-rate-hardening followed by strain-rate-softening on an increase of the Weissenberg number $W i \geq 0.3$. For these unentangled melts, the hardening was unequivocally related to the FENE effect weakened by the orientation/stretch-induced $\zeta$-reduction, and the softening, to the $\zeta$-reduction overwhelming the FENE effect. Thus, the $\zeta$-reduction, speculatively discussed for entangled melts so far, was experimentally confirmed, to the first time, for unentangled melts.

Quantitatively, the strain-rate-hardening at intermediate $W i$ was weaker and the strain-rate-softening at higher Wi was stronger for PS27 than for PtBS53, despite the similarity of their $n_{\mathrm{K}}$ values. This result suggested that the magnitude of $\zeta$-reduction depends on the chemical structure of the chains. For a further test of this dependence on the chemical structure, the FENE-PM bead-spring model was modified for the $\zeta$-reduction in an empirical way, with an 
assumption that $\zeta$ at a given time is fully determined by the chain orientation $S$ and the stretch $\lambda$ at that time and thus by the tensile stress $\tilde{\sigma}_{\mathrm{E}}\left(\sim S \lambda^{2}\right)$. This modified model was able to excellently mimic the steady state $\eta_{\mathrm{E}}$ data (given that the parameters describing the $\zeta$-reduction were properly chosen). The $\zeta$-reduction utilized in this modification was stronger for PS27 than for PtBS53, which lends support to the argument that the bulky side group of PtBS serves as a "solvent" to screen the packing interaction between the chain backbones thereby weakening the $\zeta$ reduction.

Nevertheless, the same modified model failed to describe the stress growth and relaxation data on start-up and cessation of fast elongational flow, despite its success in fitting the steady state $\eta_{\mathrm{E}}$ data. This failure suggests a possibility that transient changes of $\xi$ in the real materials (PS27 and PtBS53) during the stress growth and relaxation are delayed compared to the stress evolution. Namely, the segmental friction $\zeta$ in PS27 and PtBS53 melts at a given time appears to be not fully determined by the chain stretch/orientation (reflected in $\tilde{\sigma}_{\mathrm{E}}$ ) at that time but affected by the transient changes of the stretch/orientation, with the latter effect vanishing in the steady state (thereby allowing the model to mimic the steady state $\eta_{\mathrm{E}}$ data). This effect of the transient changes in the chain stretch/orientation on $\xi$ can be related to the local chain motion (segment motion) necessary for adjusting $\zeta$ to the transient changes of the orientation/stretch in the environment: The time necessary for this motion would/should result in the delay in the change of $\zeta$. The friction is defined only when the segment moves, so that the $\zeta$-reduction appears to be linked, by definition, to the local chain motion for achieving this adjustment. Thus, the description of the $\zeta$-reduction and the corresponding nonlinear rheology could be improved if the delay in the change of $\xi$ due to the local chain motion is properly accounted in the molecular model(s) and/or simulation. This improvement is an interesting and important subject of future work.

At the same time, we also note another possibility that the difference between the modified FENE-PM calculation and the transient state data could have partly resulted from the pre-averaging approximation for the spring stiffness and friction coefficient in the calculation. These distributions might allow less stretched (less stiffened) springs and more frictional segments to govern the transient rheological responses to reduce the difference between the calculation and the transient state data. This possibility is to be examined in future work together with the possibility of the delay in the change of $\zeta$ explained above. 


\section{Appendix A. Symbols utilized in analysis with modified FENE-PM model.}

We prefer the symbols utilized in our analysis to be identical to those in our recent papers. ${ }^{22,46}$ Our symbols are not necessarily identical to those in Ref. 34 (the original paper for the FENE-PM model). To avoid confusion, a reference table of those symbols is given below.

Table 3. List of symbols used in this study and in Ref.34.

\begin{tabular}{|l|l|l|}
\hline & \multicolumn{1}{|c|}{ This study } & \multicolumn{1}{c|}{ Ref.34 } \\
\hline number of Kuhn segments per FENE spring & $g$ & --- \\
\hline full-stretch length of FENE spring & $g l_{\mathrm{K}}$ & $Q_{0}$ \\
\hline strength of Hookean spring & $\kappa_{\mathrm{H}}$ & $H$ \\
\hline strength of FENE spring & $\kappa\left(=\kappa_{\mathrm{H}} f_{\mathrm{FENE}}\right)$ & $H Z$ \\
\hline elasticity enhancement factor due to FENE & $f_{\mathrm{FENE}}$ & $Z$ \\
\hline stress tensor of $j$-th relaxation mode & $\boldsymbol{\sigma}_{j}$ & $\boldsymbol{\tau}_{j}$ \\
\hline normalized stress tensor of $j$-th relaxation mode & $\tilde{\boldsymbol{\sigma}}_{j}\left(=\sigma_{j} / v k_{\mathrm{B}} T\right)$ & $\mathbf{T}_{j}\left(=\tau_{j} / v k_{\mathrm{B}} T\right)$ \\
\hline Rouse relaxation time of $j$-th mode (at equilibrium) & $\tau_{j}^{[\mathrm{R}]}$ & $\lambda_{j}$ \\
\hline equilibrium relaxation time of $j$-th mode in FENE-PM model & $\tau_{j}^{\mathrm{eq}}$ & --- \\
\hline anisotropic friction coefficients & $\zeta_{x}, \zeta_{y}\left(=\zeta_{x}\right), \zeta_{z}$ & --- \\
\hline anisotropic friction reduction ratio & $r_{\xi}\left(=\zeta_{\xi} / \zeta_{e q}\right)$ & --- \\
\hline
\end{tabular}




\section{Appendix B. Features of Eqs 14-16.}

B-1. Feature of eq 16: Equation 16, the key in our analysis, gives multiple solutions of the normalized FENE factor (elasticity enhancement factor) $X$. This feature of eq 16 can be easily understood if we consider the limit of $N>>1$. In this limit, the factor $a_{j} / a_{1}$ appearing in eq 16 can be replaced by $j^{2}$ (see eq 6 for the expression of $a_{j}$ ). Then, with the aid of mathematical identities, ${ }^{51}$ $\sum_{j=1}^{\infty}\left(j^{2}-\theta^{2}\right)^{-1}=1 /\left(2 \theta^{2}\right)-\pi \cot (\pi \theta) / 2 \theta$ and $\sum_{j=1}^{\infty}\left(j^{2}+\theta^{2}\right)^{-1}=-1 /\left(2 \theta^{2}\right)+\pi \operatorname{coth}(\pi \theta) / 2 \theta$, eq 16 (or eq 12) can be rewritten as

$$
X=1-\frac{1}{(b+3)(N-1)}\left(2 \tilde{\sigma}_{x x}+\tilde{\sigma}_{z z}\right)
$$

with

$$
\begin{aligned}
& \tilde{\sigma}_{x x} \equiv \sum_{j \geq 1} \tilde{\sigma}_{j, x x}=-\frac{1}{2}+\frac{1}{2}\left(\pi \sqrt{\frac{r_{x} W i}{X}}\right) \operatorname{coth}\left(\pi \sqrt{\frac{r_{x} W i}{X}}\right) \\
& \tilde{\sigma}_{z z} \equiv \sum_{j \geq 1} \tilde{\sigma}_{j, z z}=-\frac{1}{2}+\frac{1}{2}\left(\pi \sqrt{\frac{2 r_{z} W i}{X}}\right) \cot \left(\pi \sqrt{\frac{2 r_{z} W i}{X}}\right)
\end{aligned}
$$

where $r_{x}$ and $r_{z}$ indicate the $\zeta$-reduction ratios in $x$ and $z$ directions. Because the $\left(\pi \sqrt{2 r_{z} W i / X}\right) \cot \left(\pi \sqrt{2 r_{z} W i / X}\right)$ factor included in $\tilde{\sigma}_{z z}$ (eq 35) oscillates with an infinitely large amplitude on an increase of Wi, eq 33 (eq 16) gives multiple solutions of $X$. In the analysis presented in the main text, we numerically solved eq 16 and chose the minimum value of the solutions of $X$ satisfying a physical requirement that $X$ never decreases with increasing Wi (namely, the chain is always stretched and stiffened more significantly under faster flow).

This feature of eq 33 (eq 16) in turn means that $X$ for large $W i$ is essentially determined by the $\tilde{\sigma}_{z z}$ term that includes $r_{\mathrm{z}}$ but not $r_{\mathrm{x}}$. (For large $W i$, this term overwhelms the $\tilde{\sigma}_{x x}$ term in eq 33.) Namely, for large $W i, X$ is insensitive to $r_{x}$ and mostly determined by $r_{z}$ (or, by the product $r_{z} W i$ ).

B-2. Feature of eqs 14 and 15: As explained in the main text, our analysis gave almost indistinguishable normalized tensile stress $\tilde{\sigma}_{\mathrm{E}}$ for the two extreme cases of fully anisotropic and isotropic $\zeta$-reduction. This result, reflecting the features of eqs 14 and 15, can be qualitatively understood again by considering the limit of $N>1$. In this limit, $\tilde{\sigma}_{\mathrm{E}}$ is expressed as $\tilde{\sigma}_{\mathrm{E}}=\tilde{\sigma}_{x x}-\tilde{\sigma}_{z z}$ (eq 13) with $\tilde{\sigma}_{x x}$ and $\tilde{\sigma}_{z z}$ being given by eqs 34 and 35, respectively. As explained above, $\tilde{\sigma}_{z z}$ overwhelms $\tilde{\sigma}_{x x}$ for large $W i$, so that $\tilde{\sigma}_{\mathrm{E}}$ almost coincides with $-\tilde{\sigma}_{z z}$ being 
insensitive to $r_{x}$ (note that $X$ appearing in eq 35 is insensitive to $r_{x}$ for large $W i$ ). In contrast, for small Wi, $\tilde{\sigma}_{x x} \sim r_{x}\left(\pi^{2} / 4 X\right) W i$ and $-\tilde{\sigma}_{z z} \sim r_{z}\left(\pi^{2} / 2 X\right) W i$ (cf. eqs 34 and 35) so that $\tilde{\sigma}_{\mathrm{E}}$ depends on both $r_{x}$ and $r_{z}$. Nevertheless, $r_{x}$ and $r_{z}$ are close to unity for small $W i$, and this dependence of $\tilde{\sigma}_{\mathrm{E}}$ is minor in any case.

Thus, eqs 14 and 15 combined with eq 16 (eqs 34 and 35 with eq 33) give $\tilde{\sigma}_{\mathrm{E}}$ being insensitive to $r_{x}$ and mostly determined by $r_{z}$ in the entire range of Wi. This feature of eqs 14 and 15 resulted in almost indistinguishable $\tilde{\sigma}_{\mathrm{E}}$ obtained from our analysis for the two extreme cases of fully anisotropic and isotropic $\zeta$-reduction with the parameter values shown in Table 2.

The coincidence for the two extreme cases is not limited to the steady flow state (where eqs 14-16 are valid and the coincidence is noted in Figure 5) but is confirmed also in the transient state. As an example, Figure 11 shows growth of the normalized FENE factor $X$ and decay of the $\zeta$-reduction ratio $r_{z}\left(=\zeta_{z} / \zeta_{\text {eq }}\right)$ with time for the two extreme cases calculated for PS27 on start-up flow at $W i=21.5\left(\dot{\varepsilon}=0.5 \mathrm{~s}^{-1}\right)$. The corresponding growth of the normalized stress components, $\tilde{\sigma}_{x x}$ and $-\tilde{\sigma}_{z z}$, is shown in Figure 12.
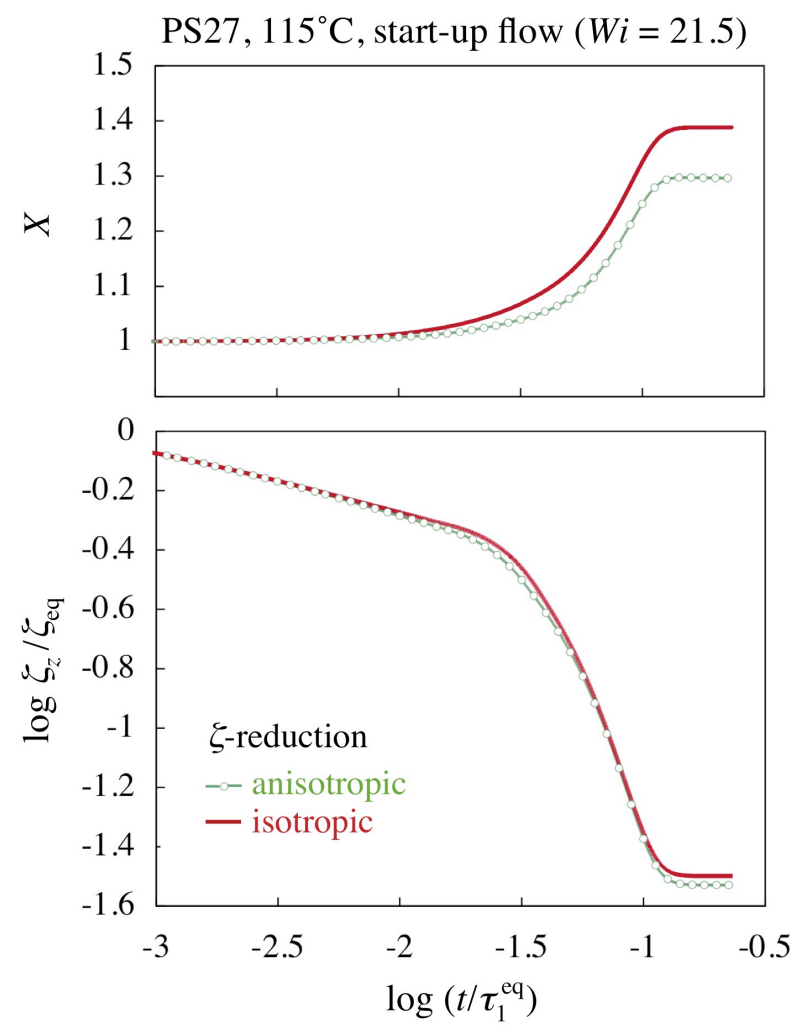

Figure 11. Growth of $X$ and decay of $r_{\mathrm{z}}\left(=\zeta_{z} / \zeta_{\mathrm{eq}}\right.$ of PS27 calculated for start-up flow $(W i=21.5)$ for two extreme cases of anisotropic and isotropic $\zeta$-reduction. 


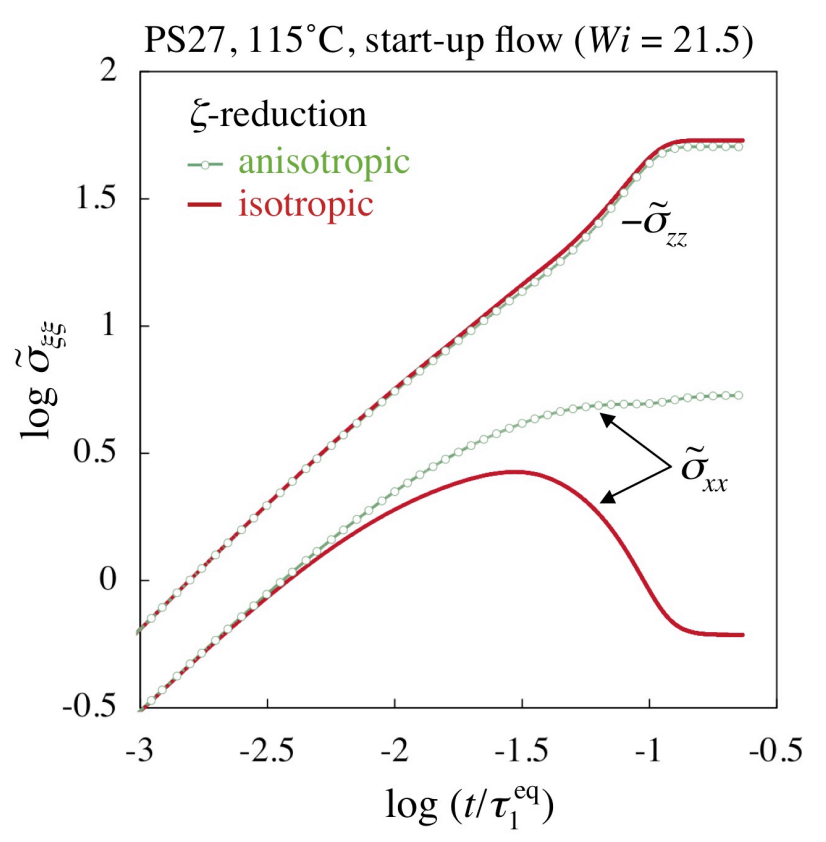

Figure 12. Growth of normalized stress components of PS27 calculated for start-up flow $(W i=21.5)$ for two extreme cases of anisotropic and isotropic $\zeta$-reduction.

For the parameters given in Table 2, Figure 11 demonstrates that $X$ grows (the chain is stretched) with time a little less significantly in the anisotropic case $\left(r_{z}<r_{x}=1\right)$ than in the isotropic case $\left(r_{z}=r_{x}<1\right)$ but $r_{z}$ is almost indistinguishable for the two cases (despite the moderate difference of $X$ ). Correspondingly, $\tilde{\sigma}_{x x}$ exhibits a clear difference at long $t>\tau_{1}^{\mathrm{eq}} / 100$ (significant overshoot observed only for the case of isotropic $\zeta$-reduction), as noted in Figure 12. Nevertheless, $-\tilde{\sigma}_{z z}$ is almost identical for the two cases and overwhelms $\tilde{\sigma}_{x x}$. Consequently, the normalized tensile stress $\tilde{\sigma}_{\mathrm{E}}\left(=\tilde{\sigma}_{x x}-\tilde{\sigma}_{z z}\right)$ is almost the same in those cases (as explained for Figure 3), which allows us to examine the magnitudes of $\zeta$-reduction in PS27 and PtBS53 melts with no ambiguity, despite the lack of information for the anisotropy of $\xi$-reduction. (At the same time, this lack of ambiguity means that the anisotropy cannot be resolved from comparison of the current calculation and data.) 


\section{Appendix C. Elongational viscosity calculated from FENE-PM model for various $N$ values.}

Figure 13 shows the $\eta_{\mathrm{E}} / \eta_{\mathrm{E} 0}$ ratio calculated from the FENE-PM model without $\zeta$-reduction. The calculation was conducted in a way explained for eqs 14-17 but by setting $r_{x}$ and $r_{\mathrm{z}}$ therein to be unity (i.e., no $\zeta$-reduction). The number of Kuhn segments per chain is fixed at $n_{\mathrm{K}}=30$, and the number of beads per chain, $N$, is varied from 2 to $30 . \quad(N=2$ corresponds to the FENE-dumbbell model. ${ }^{35,42,46}$ ) The calculated $\eta_{\mathrm{E}} / \eta_{\mathrm{E} 0}$ ratio does not significantly change with our choice of $N$, in particular in the range of $3 \leq N \leq 16$. This insensitivity to the choice of $N$ was noted also for the FENE-PM model modified for the $\zeta$-reduction.

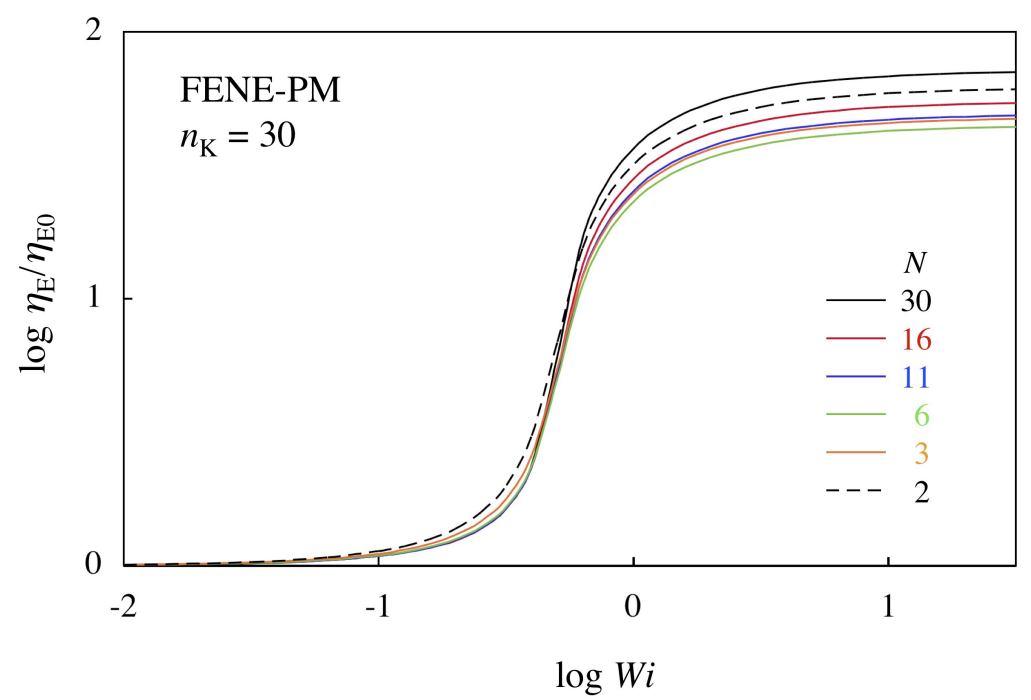

Figure 13. Normalized steady state elongational viscosity $\left(\eta_{\mathrm{E}} / \eta_{\mathrm{E} 0}\right.$ ratio $)$ calculated from the FENE-PM model without $\zeta$-reduction. The $\eta_{\mathrm{E}} / \eta_{\mathrm{E} 0}$ ratio is plotted against the Weissenberg number. The number of Kuhn segments per chain is fixed at $n_{\mathrm{K}}=$ 30 , and the number of beads per chain, $N$, is varied from 2 to 30 . 


\section{Appendix D. Comparison with $\zeta$-Reduction of Entangled Melts Reported in Literature:}

It is informative to compare the $\zeta$-reduction ratio $r_{z}\left(=\zeta_{z} / \zeta_{\mathrm{eq}}\right)$ that excellently mimicked the $\eta_{\mathrm{E}} / \eta_{\mathrm{E} 0}$ data of our unentangled PS27 melt (Figure 5) with the ratio for entangled PS melts reported in literature..$^{21,22}$ This Appendix is devoted for this comparison.

Hassager and coworkers ${ }^{20}$ reported elongational stress relaxation behavior of entangled PS145 melt $(M=145 \mathrm{k})$ on cessation of flow at several $\dot{\varepsilon}$. Yaoita and coworkers ${ }^{22}$ decomposed those data into exponentially decaying modes from long $t$ to short $t$ (via so-called procedure $\mathrm{X}^{52}$ ) to evaluate the decay time ( $t$-independent constant) of those modes, $\tau_{j}^{[\mathrm{X}]}$ for $j=1-5$. This $\tau_{j}^{[\mathrm{X}]}$ differs from $\tau_{j}$ of the relaxation modes analyzed in the FENE-PM model with/without $\zeta$-reduction: $\tau_{j}$ changes with $t$ (for example, during the stress relaxation process, $\tau_{j}$ increases with $t$ because of the decrease of chain stretch and the increase of $\zeta$ with $t$ ). Nevertheless, a decrease of $\tau_{j}^{[\mathrm{X}]}$ for fast modes (for large $j$ ) with $\dot{\varepsilon}$, found for PS145k, ${ }^{22}$ characterizes the acceleration of the relaxation just after the flow cessation thereby reflecting the FENE effect and $\xi$-reduction for entangled PS145 under the flow. Thus, it is informative to analyze the stress relaxation data of our unentangled PS27 melt (Figure 6) with the method of Yaoita and coworkers. ${ }^{22}$

We made this analysis, and compared $\tau_{j}^{[\mathrm{X}]} / \tau_{1}^{\mathrm{eq}}$ ratios for the PS27 and PS145 melts because the longest Rouse (or FENE-PM) relaxation times measured for those melts at equilibrium, $\tau_{1}^{\mathrm{eq}}$, were different due to the differences in their molecular weights and measurement temperatures. The results of comparison are presented in Figure 14 where the $\tau_{j}^{[\mathrm{X}]} / \tau_{1}^{\mathrm{eq}}$ ratio is double-logarithmically plotted against the index $j$ of the exponentially decaying mode.

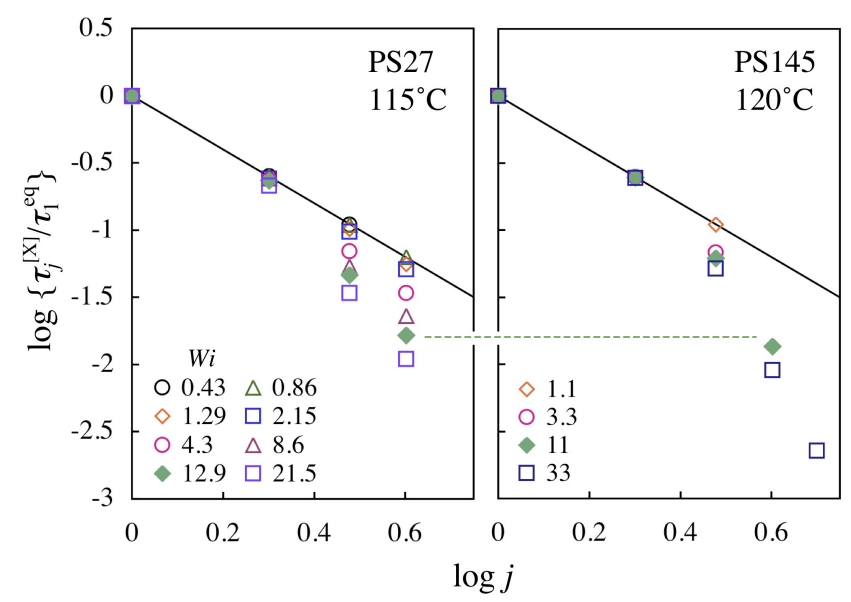

Figure 14. Comparison of $\tau_{j}^{[\mathrm{X}]} / \tau_{1}^{\mathrm{eq}}$ ratio obtained for unentangled PS27 melt (this work; left panel) and entangled PS145 reported by Yaoita and coworkers ${ }^{22}$ (right panel). The ratios obtained for several values of Rouse-based (or FENE-PM-based) Weissenberg number $W i$ are plotted against index $j$ of the exponentially decaying mode of stress. 
As noted in Figure 14, the relaxation of both PS27 and PS145 is more strongly accelerated on cessation of faster flow. Comparing the $\tau_{j}^{[\mathrm{X}]} / \tau_{1}^{\mathrm{eq}}$ ratios in left and right panels for similar values of the Rouse-based (or FENE-PM-based) Weissenberg numbers Wi, we note that the magnitude of acceleration is similar for PS27 and PS145; see green diamonds, for example. This similarity suggests that the $\tau_{j}^{[\mathrm{X}]} / \tau_{1}^{\mathrm{eq}}$ ratios of the unentangled PS27 and entangled PS145 melts reflect the same type of fast nonlinear dynamics just after the flow cessation. However, the acceleration reflects not only the $\zeta$-reduction but also the FENE effect. Yaoita and coworkers ${ }^{22}$ formulated an empirical equation describing the observed acceleration (data in the right panel of Figure 14) as a function of the tensile stress $\sigma_{\mathrm{E}}$, incorporated this equation in primitive chain $(\mathrm{PCN})$ simulation for entangled chains adopting the pre-averaged FENE effect described by eq 5, and evaluated the isotropic $\zeta$-reduction ratio $r=\xi / \zeta_{\mathrm{eq}}\left(=r_{x}=r_{z}\right.$ in the definition in this study) that was consistent with $\sigma_{\mathrm{E}}$ and the FENE factor deduced from the simulation. This method of evaluation cannot be applied to our unentangled PS27 melt. Nevertheless, it is interesting to compare the ratio $r$ reported by Yaoita and coworkers ${ }^{22}$ with the ratio $r_{\mathrm{z}}$ for PS27 obtained in this study.

Ianniruberto and coworkers ${ }^{21}$ analyzed literature data of $\sigma_{\mathrm{E}}$ and the birefringence $\Delta n$ of entangled PS206 melt $(M=206 \mathrm{k})$ reported by Luap and coworkers ${ }^{53}$ to evaluate the isotropic $\zeta$-reduction ratio, $r=\xi / \zeta_{\text {eq }}$, under strong elongational flow giving large $\sigma_{\mathrm{E}}(>1 \mathrm{MPa})$. For such large $\sigma_{\mathrm{E}}$, the PS chains are highly oriented and stretched. Thus, Ianniruberto and coworkers ${ }^{21}$ assumed that the entanglement segments in the chain are fully oriented, and evaluated the ratio $r$ from analysis of $\sigma_{\mathrm{E}}$ and $\Delta n$ for such a fully oriented chain. This method of evaluation cannot be applied to our PS27 melt (because of lack of the $\Delta n$ data), but it is again interesting to compare the reported $r$ with our $r_{\mathrm{z}}$ for PS27.

Figure 15 compares the isotropic $\zeta / \zeta_{\text {eq }}$ ratios of entangled PS melts reported by Yaoita and coworkers $^{22}$ and by Ianniruberto and coworkers ${ }^{21}$ with the $\zeta_{z} / \zeta_{\text {eq }}$ ratio of unentangled PS27 melt obtained in this study. The double-linear format of the plots of $\zeta / \zeta_{\text {eq }}$ against $\sigma_{\mathrm{E}}$, presented in Figure 2 of Ref.21 in the range of $\sigma_{\mathrm{E}} \geq 1.5 \mathrm{MPa}$, is converted to the double-logarithmic format in our Figure 15 (thick blue curve). In Figure 11 of Ref. 22, the $\zeta / \zeta_{\text {eq }}$ ratio is plotted against a stretch/orientation parameter $F_{\text {so }}=\tilde{\lambda}^{2} \bar{S}$, where $\tilde{\lambda}$ represents the stretch ratio of entanglement strand reduced by its maximum stretch ratio and $\bar{S}$ denotes the orientational anisotropy of the strand (averaged with a solvent, if any). This $F_{\text {so }}$ is related to $\sigma_{\mathrm{E}}$ as $F_{\text {so }}=\sigma_{\mathrm{E}} \phi_{\mathrm{p}} /\left\{3 G_{\mathrm{e}} n_{0} f_{\mathrm{FENE}}\right\}$ with 
$\phi_{\mathrm{p}}, n_{0}$, and $G_{\mathrm{e}}$ being the volume fraction of the polymer (= 1 in melt), number of Kuhn segments per entanglement strand at equilibrium, and the entanglement plateau modulus, respectively. Utilizing this relationship, we converted Figure 11 of Ref. 22 into our $\zeta / \zeta_{\text {eq }} v s \sigma_{\mathrm{E}}$ plots in Figure 15 (thick orange curve). The $\zeta_{z} / \zeta_{\text {eq }}$ ratio of unentangled PS27, giving the modified FENE-PM calculation that excellently mimicked the $\eta_{\mathrm{E}} / \eta_{\mathrm{E} 0}$ data (Figure 5), is shown with small circle and thin curve for the two extreme cases of fully anisotropic and isotropic $\zeta$-reduction, respectively.

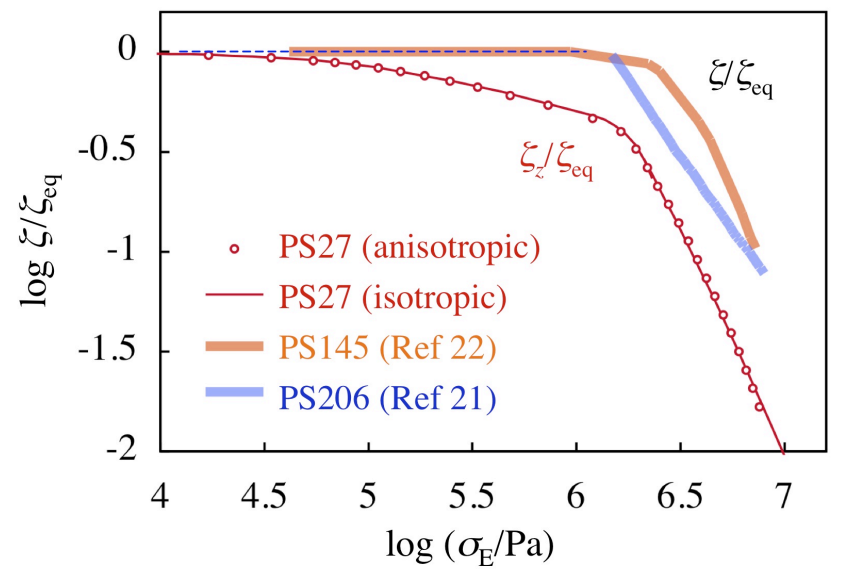

Figure 15. Comparison of friction reduction ratios for unentangled PS 27 melt (evaluated in this work) and the ratios for entangled PS melts reported in literatures. ${ }^{21,22}$ The ratios are double-logarithmically plotted against the tensile stress $\sigma_{\mathrm{E}}$.

Figure 15 demonstrates that the $\zeta / \zeta_{\text {eq }}$ ratio of entangled PS reported in literature ${ }^{21,22}$ (thick curves) is moderately larger ( $\zeta$-reduction is weaker), by a factor of $2-3$, than the $\zeta_{z} / \zeta_{\text {eq }}$ ratio of PS27 shown with the small circle and thin curve. This difference is a little puzzling because the $\zeta$-reduction is believed to be a local event irrelevant to the entanglement. The reported isotropic $\zeta / \zeta_{\text {eq }} \operatorname{ratio}^{21,22}$ could be a sort of average between $\zeta_{z} / \zeta_{\mathrm{eq}}$ and $\zeta_{x} / \zeta_{\mathrm{eq}}$ in the realistic case of anisotropic $\zeta$-reduction for which $\zeta_{z} / \zeta_{\text {eq }}$ is smaller than $\zeta_{x} / \zeta_{\text {eq }}$ in general. This averaging feature of the $\zeta / \zeta_{\text {eq }}$ ratio could have resulted in the moderate difference explained above. At the same time, we cannot rule out a possibility that some entanglement effect was missing in the literature analysis ${ }^{21,22}$ of the $\Delta n$ and/or $\sigma_{\mathrm{E}}$ data and the resulting $\zeta / \zeta_{\mathrm{eq}}$ ratio was not of purely frictional nature but contributed from entanglement nonlinearities. This potentially remaining entanglement contribution might have resulted in the above difference. Thus, the difference seen in Figure 15 deserves further attention in future work.

Finally, we also note the other possibility that is related to pre-averaging approximation for the 
spring stiffness and the segment friction in our modified PENE-PM analysis. In actual melts, each chain should have distributions of the stiffness and friction along its backbone. If this distribution is very broad and the pre-averaging in our analysis weighs too heavily on less frictional segments connected to more stretched springs than treated in literature, ${ }^{21,22}$ the resulting friction coefficient could be smaller (friction reduction could be stronger) in our analysis than in literature analyses, as seen in Figure 15. This possibility is to be also examined in future work. 


\section{Appendix E. Acceleration of Stress Relaxation in Modified FENE-PM Model due to FENE Effect and Friction Reduction}

It is informative to compare contributions of the FENE effect and $\zeta$-reduction to the acceleration of the stress relaxation after cessation of flow. For this purpose, we utilized the modified FENE-PM model (eqs 28-32 with the parameters specified in Table 2) to calculate the rubbery component of the normalized tensile stress, $\tilde{\sigma}_{\mathrm{E}, \mathrm{r}}^{-}(t)=\sigma_{\mathrm{E}, \mathrm{r}}^{-}(t) / v k_{\mathrm{B}} T$, after cessation of flow at a strain of $\varepsilon=2.5$ (the experimental protocol for PS27 and PtBS53 melts). For the fastest flow examined $\left(\dot{\varepsilon}=0.5 \mathrm{~s}^{-1}\right)$, the results are shown in Figure 16 with the red asterisk and red curve for the cases of fully anisotropic and isotropic $\zeta$-reduction. As a reference specifying the acceleration due only to the FENE effect in the modified FENE-PM model, we calculated $\tilde{\sigma}_{\mathrm{E}, \mathrm{r}}^{-}(t)$ from the original FENE-PM model without $\zeta$-reduction but for the initial condition (eq 28) identical to that for the modified model; black asterisk and black curve show the results obtained from the initial condition identical to that for the red asterisk and red curve. For both modified and original FENE-PM calculations, $\tilde{\sigma}_{\mathrm{E}, \mathrm{r}}^{-}(t)$ is normalized by its initial value $\tilde{\sigma}_{\mathrm{E}, \mathrm{r}}^{-}(0)$ and plotted against time $t$ after the flow cessation.

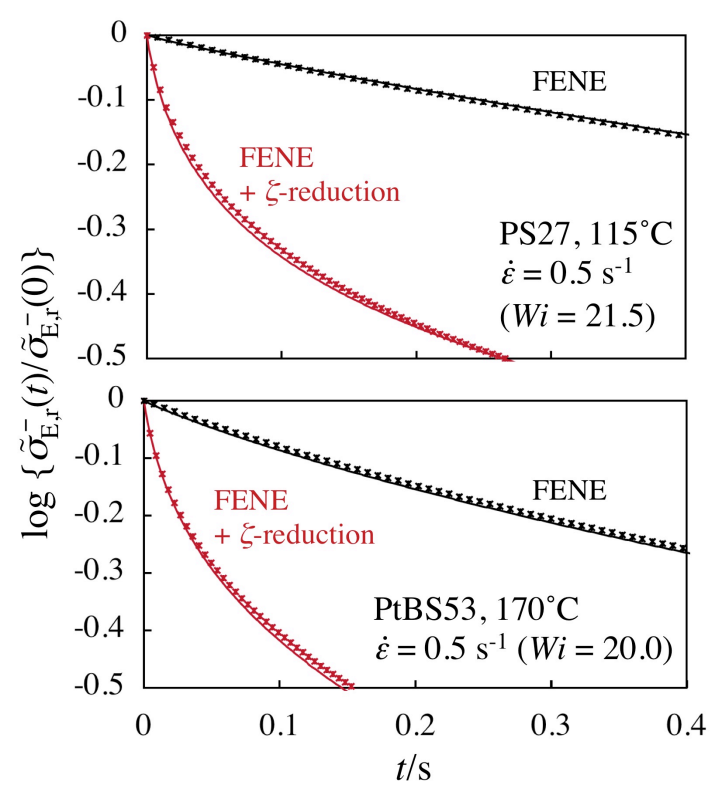

Figure 16. Relaxation after cessation of flow at $\dot{\varepsilon}=0.5 \mathrm{~s}^{-1}$ calculated from modified FENE-PM model for PS27 and PtBS53; red asterisk and red curve show the results for the extreme cases of fully anisotropic and isotropic $\zeta$-reduction. The relaxation calculated from the original FENE-PM model without $\zeta$-reduction but for the initial condition (eq 28) identical to that for the modified model are shown with black asterisk and black curve. 
Figure 16 clearly demonstrates that the stress relaxation after flow at $\dot{\varepsilon}=0.5 \mathrm{~s}^{-1}$ is much faster for the modified FENE-PM model than for the original FENE-PM model, both starting from the same initial condition. Thus, after cessation of such fast flow, the $\xi$-reduction accelerates the relaxation much more strongly compared to the FENE effect. This difference in the acceleration due to the $\zeta$-reduction and FENE effect can be most clearly examined for the normalized initial relaxation rate of the rubbery stress component (NIRR) defined by

$$
\mathrm{NIRR}=-\left[\frac{\mathrm{d} \tilde{\sigma}_{\mathrm{E}, \mathrm{r}}^{-}(t) / \mathrm{d} t}{\tilde{\sigma}_{\mathrm{E}, \mathrm{r}}^{-}(t)}\right]_{t \rightarrow 0}
$$

As shown in Figure 17, NIRR of the modified FENE-PM model (red circle and curve) is close to NIRR of the original model (black circle and curve) at the Weissenberg number $W i<1$, indicating that the acceleration in the modified model after cessation of slow flow is mostly due to the FENE effect. However, NIRR becomes much larger for the modified model on an increase of Wi > 1: In particular, for the highest Wi examined (that corresponds to $\dot{\varepsilon}=0.5 \mathrm{~s}^{-1}$ ), the acceleration due to $\zeta$-reduction is stronger than that due to the FENE effect by a factor of $\sim 30$ and $\sim 20$ for PS27 and PtBS53, respectively. Thus, in the modified FENE-PM model, the initial acceleration of relaxation after cessation of fast flow is dominated by $\zeta$-reduction. The acceleration in actual PS27 and PtBS53 melts on cessation of fast flow would have been similarly dominated by $\zeta$-reduction.

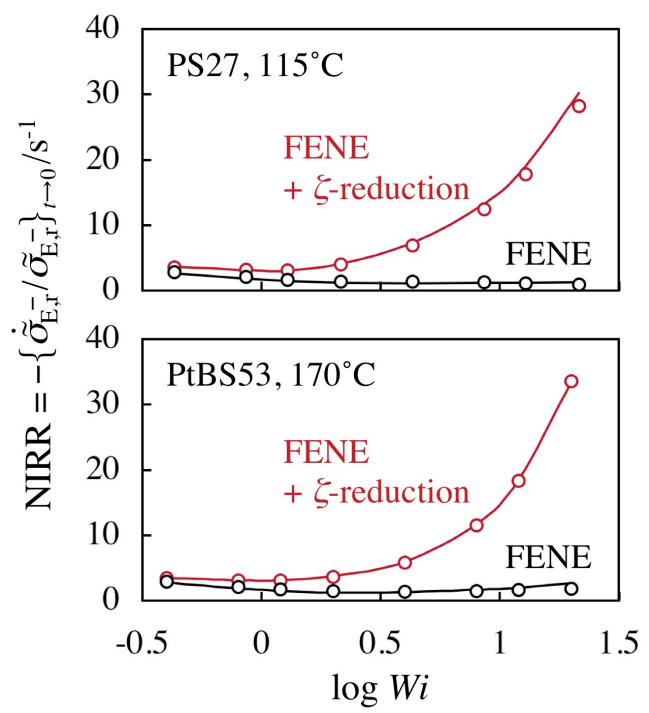

Figure 17. Changes of the normalized initial relaxation rate of the rubbery stress component with Weissenberg number Wi calculated for PS27 and PtBS53. Circle and curve indicate the rate obtained from the initial condition after stress growth with fully anisotropic and isotropic $\zeta$-reduction, and their color specifies the condition during the relaxation, red and black with and without the $\zeta$-reduction (modified and original FENE-PM calculations). 


\section{Acknowledgement:}

This work was partly supported by the Grant-in-Aid for Scientific Research (C) from JSPS, Japan (grant No. 15K05519), the Collaborative Research Program of ICR, Kyoto University (Grant No. 2018-77), and Collaborative Research Program of Kyoto University Research Coordination Alliance. We also acknowledge with thanks a possibility suggested by a reviewer of this paper that the difference between our calculation and the transient rheological data may have partly resulted from the pre-averaging of spring stiffness and segmental friction in our analysis. His/her suggestion enabled us to improve the discussion in this paper.

\section{Supporting Information:}

A. Analysis of Modified FENE-PM Model for Stress Growth after Start-up of Flow, B. Analysis of Modified FENE-PM Model for Stress Relaxation after Cessation of Flow.

These materials are available free of charge on the ACS Publications website.

\section{References and Notes.}

1. Ferry, J. D. Viscoelastic Properties of Polymers, Wiley: New York, 1982.

2. Graessley, W. W. "The Entanglement Concept in Polymer Rheology" Adv. Polym. Sci. 1974, 16, 1-179.

3. Larson, R. G. The Structure and Rheology of Complex Fluids, Oxford University Press: New York, 1999.

4. Graessley, W. W. Polymeric Liquids \& Network: Dynamics and Rheology, Garland Science: New York, 2008.

5. Watanabe, H. "Viscoelasticity and Dynamics of Entangled Polymer" Prog. Polym. Sci. 1999, 24, 1253-1403.

6. McLeish, T. C. B "Tube theory of entangled polymer dynamics" Adv. Phys. 2002, 51, $1379-1527$.

7. Baumgaertel, M.; Schausberger, A.; Winter, H. H. "The Relaxation of Polymers with Linear Flexible Chains of Uniform Length" Rheol. Acta 1990, 29, 400-408. 
8. Fetters, L. J.; Lohse, D. J.; Milner, S. T.; Graessley, W. W. "Packing Length Influence in Linear Polymer Melts on the Entanglement, Critical, and Reptation Molecular Weights" Macromolecules 1999, 32, 6847-6851.

9. Inoue, T.; Okamoto, H.; Osaki, K. "Birefringence of Amorphous Polymers. 1. Dynamic Measurement on Polystyrene" Macromolecules 1991, 24, 5670-5675.

10. Okamoto, H.; Inoue, T.; Osaki, K. "Viscoelasticity and Birefringence of Polyisobutylene" J. Polym. Sci. B: Polym. Phys. 1995, 33, 1409-1416.

11. Inoue, T.; Matsui, H.; Osaki, K. "Molecular Origin of Viscoelasticity and Chain Orientation of Glassy Polymers" Rheol. Acta 1997, 36, 239-244.

12. Matsumiya, Y.; Kumazawa, K.; Nagao, M.; Urakawa, O.; Watanabe, H. "Dielectric Relaxation of Monodisperse Linear Polyisoprene: Contribution of Constraint Release" Macromolecules 2013, 46, 6067-6080.

13. Huang, Q.; Hengeller, L.; Alvarez, N. J.; Hassager O. "Bridging the Gap between Polymer Melts and Solutions in Extensional Rheology" Macromolecules 2015, 48, 4158-4163

14. Costanzo, S.; Huang, Q.; Ianniruberto, G.; Marrucci, G.; Hassager, O.; Vlassopoulos, D. "Shear and Extensional Rheology of Polystyrene Melts and Solutions with the Same Number of Entanglements" Macromolecules 2016, 49, 3925-3935.

15. Shivokhin, M. E.; Read, D.; Kouloumasis, D.; Kocen, R.; Zhuge, F.; Bailly, C.; Hadjichristidis, N.; Likhtman, A. E., "Understanding Effect of Constraint Release Environment on End-to-End Vector Relaxation of Linear Polymer Chains" Macromolecules 2017, 50, 4501-4523.

16. Bhattacharjee, P. K.; Oberhauser, J. P.; McKinley, G. H.; Leal, L. J.; Sridhar, T. "Extensional Rheometry of Entangled Solutions" Macromolecules 2002, 35, 10131-10148.

17. Bhattacharjee, P. K.; Nguyen, D. A.; McKinley, G. H.; Sridhar, T. "Extensional stress growth and stress relaxation in entangled polymer solutions" J. Rheol. 2003, 47, 269-290.

18. Bach, A.; Almdal, K.; Rasmussen, H. K.; Hassager, O. "Elongational Viscosity of Narrow Molar Mass Distribution Polystyrene" Macromolecules 2003, 36, 5174-5179.

19. Nielsen, J. K.; Rasmussen, H. K.; Hassager, O.; McKinley, G. H. J. "Elongational viscosity of monodisperse and bidisperse polystyrene melts" J. Rheol. 2006, 50, 453-476.

20. Nielsen, J. K.; Rasmussen, H. K.; Hassager, O. "Stress relaxation of narrow molar mass distribution polystyrene following uniaxial extension" J. Rheol. 2008, 52, 885-899. 
21. Ianniruberto, G.; Brasiello, A.; Marrucci, G. "Simulations of Fast Shear Flows of PS Oligomers Confirm Monomeric Friction Reduction in Fast Elongational Flows of Monodisperse PS Melts As Indicated by Rheooptical Data" Macromolecules 2012, 45, 8058-8066. (The idea of friction reduction was first presented by Ianniruberto, Brasiello, and Marrucci in 2011 at 7th Annual European Rheology Conference; cf. p.61 in the Abstract Book.)

22. Yaoita, T.; Isaki, T.; Masubuchi, Y.; Watanabe, H.; Ianniruberto, G.; Marrucci, G. "Primitive Chain Network Simulation of Elongational Flows of Entangled Linear Chains: Stretch/Orientation-induced Reduction of Monomeric Friction" Macromolecules 2012, 45, 2773-2782.

23. Huang, Q.; Alvarez, N. J.; Matsumiya, Y.; Rasmussen, H. K.; Watanabe, H.; Hassager, O. "Extensional Rheology of Entangled Polystyrene Solutions Suggests Importance of Nematic Interactions" ACS Macro Lett. 2013, 2, 741-744.

24. Huang, Q.; Mednova, O.; Rasmussen, H. K.; Alvarez, N. J.; Skov, A. L.; Almdal, K.; Hassager, O. “Concentrated Polymer Solutions are Different from Melts: Role of Entanglement Molecular Weight" Macromolecules 2013, 46, 5026-5035.

25. Masubuchi, Y.; Matsumiya, Y.; Watanabe, H. “Test of Orientation/Stretch-Induced Reduction of Friction via Primitive Chain Network Simulations for Polystyrene, Polyisoprene, and Poly(n-butyl acrylate)" Macromolecules 2014, 47, 6768-6775.

26. Sridhar, T.; Acharya, M.; Nguyen, D.; Bhattacharjee, P. K. Macromolecules "On the Extensional Rheology of Polymer Melts and Concentrated Solutions” 2014, 47, 379-386.

27. Marrucci, G. "Dynamics of entanglements: A nonlinear model consistent with the Cox-Merz rule" J. Non-Newtonian Fluid Mech. 1996, 62, 279-289.

28. Marrucci, G.; Ianniruberto, G. "Interchain Pressure Effect in Extensional Flows of Entangled Polymer Melts" Macromolecules 2004, 37, 3934-3942.

29. Wagner, M. H.; Kheirandish, S.; Hassager, O. "Quantitative prediction of transient and steady-state elongational viscosity of nearly monodisperse polystyrene melts" J. Rheol. 2005, 49, 1317-1327.

30. Desai, P. S.; Larson, R. G. "Constitutive model that shows extension thickening for entangled solutions and extension thinning for melts" J. Rheol. 2014, 58, 255-279.

31. Yaoita, T.; Masubuchi, Y.; Watanabe, H. "Concept of Stretch/Orientation-Induced Friction 
reduction Tested with a Simple Molecular Constitutive equation" Nihon Reoroji Gakkaishi (J. Soc. Rheol. Japan) 2014, 42, 207-213.

32. Masubuchi, Y.; Yaoita, T.; Matsumiya, Y.; Watanabe, H.; Ianniruberto, G.; Marrucci, G. "Stretch/orientation Induced Acceleration in Stress Relaxation in Coarse-Grained Molecular Dynamic Simulations" Nihon Reoroji Gakkaishi (J. Soc. Rheol. Japan) 2013, 41, 35-37.

33. López-Barrón, C. R.; Zhou, H.; Younker, J. M.; Mann, J. A. "Molecular Structure, Chain Dimensions, and Linear Rheology of Poly(4-vinylbiphenyl)" Macromolecules 2017, 50, 9048-9057.

34. Wedgewood, L. E.; Ostrov, D. N.; Bird, R. B. “A finite extensible bead-spring chain mode for dilute polymer solutions" J. Non-Newtonian Fluid Mech. 1991, 40, 119-139.

35. Bird, R. B.; Hassager, O.; Armstrong, R. C.; Curtis, C. F. Dynamics of Polymeric Liquids. Volume 2. Kinetic Theory, Wiley: New York, 1977.

36. Doi, Y.; Takano, A.; Takahashi, Y.; Matsushita, Y. "Melt Rheology of Tadpole-Shaped Polystyrenes" Macromolecules 2015, 48, 8667-8674.

37. Chen, Q.; Matsumiya, Y.; Masubuchi, Y.; Watanabe, H.; Inoue, T. "Dynamics of Polyisoprene-Poly(p-tert-butyl styrene) Diblock Copolymer in Disordered State" Macromolecules 2011, 44, 1585-1602.

38. Fetters, L. J.; Lohse, D. J.; Colby, R. H. "Chain Dimensions and Entanglement Spacings", in Physical Properties of Polymer Handbook, 2nd ed.; Mark, J. E. Ed.; Springer: Berlin, 2007; Chapter 25.

39. (a) The Kuhn segment molecular weight $M_{\mathrm{K}}$ was evaluated from the data for the characteristic ratio, $C_{\infty} \cong 10.8$ for $\mathrm{PS}^{39 \mathrm{~b}}$ and $C_{\infty} \cong 13.0$ for $\mathrm{PtBS},{ }^{39 b, 39 \mathrm{c}}$ and the unperturbed end-to-end distance, $\left\langle R^{2}\right\rangle_{0}^{1 / 2}=6.6_{1} \times 10^{-2} M^{1 / 2}(\mathrm{~nm})$ for $\mathrm{PS}^{38}$ and $\left\langle R^{2}\right\rangle_{0}^{1 / 2}=6.0_{1} \times 10^{-2} M^{1 / 2}(\mathrm{~nm})$ for PtBS. ${ }^{38}$ (b) Kurata, M.; Tsunashima, Y. "Viscosity-Molecular Weight Relationships and Unperturbed Dimensions of Linear Chain Molecules", in Polymer Handbook, 3rd ed., Brandrup J. \& Immergut E. H. Ed., Wiley: New York, 1989, Chapter VII. (c) Mays, J. W.; Ferry, W. M.; Hadjichristidis, N.; Funk, W. G.; Fetters, L. J. "Solution properties and chain flexibility of poly(p-tert-butylstyrene)" Polymer 1986, 27, 129-132.

40. Inoue, T.; Matsui, H.; Osaki, K. "Molecular origin of viscoelasticity and chain orientation of glassy polymers" Rheol. Acta 1997, 36, 239-244. 
41. Doyle, P. S., Shaqfeh, E. S. G.; McKinley, G. H.; Spiegelberg, S. H. "Relaxation of dilute polymer solutions following extensional flow", J. Non-Newtonian Fluid Mech. 1998, 76, 79-110.

42. Larson. R. "The rheology of dilute solutions of flexible polymers: Progress and problems" $J$. Rheol. 2005, 49, 1-70.

43. Schroeder, C. K. "Single polymer dynamics for molecular rheology" J. Rheol. 2018, 62, 371-403.

44. Ianniruberto, G. "Extensional Flows of Solutions of Entangled Polymers Confirm Reduction of Friction Coefficient" Macromolecules 2015, 48, 6306-6312.

45. Herrchen, M.; Öttinger, H. C. "A detailed comparison of various FENE dumbbell models" $J$. Non-Newtonian Fluid Mech. 1997, 68 17-42.

46. Watanabe, H.; Matsumiya, Y. "Revisit the Elongational Viscosity of FENE Dumbbell Model" Nihon Reoroji Gakkaishi (J. Soc. Rheol. Japan) 2017, 45, 185-190.

47. Hunt, T. A.; Todd, B. D. "Diffusion of linear polymer melts in shear and elongational flows" J. Chem. Phys. 2009, 131, 054904, 1-12.

48. Okamoto, H.; Inoue, T.; Osaki, K. "Birefringence of Amorphous Polymers. 4. Large Deformation of Polystyrene near Its Glass Transition Temperature” Macromolecules 1992, 25, 3413-3415.

49. Inoue, T.; Ryu, D.-S.; Osaki, K. "A Rheo-Optical Study on Polystyrene under Large Tensile Deformation around the Glass Transition Temperature" Macromolecules 1998, 31, 6977-6983.

50. Matsumiya, Y.: Watanabe, H.; Inoue, T.; Osaki, K.; Yao, M.-L. "Rheo-Dielectric Behavior of Oligostyrene and Polyisoprene" Macromolecules 1998, 31, 7973-7975.

51. Moriguchi, S.; Udagawa, K.; Hitotsumatsu, S. Mathematical Formula, Iwanami: Tokyo, 1986.

52. Tobolsky, A. V.; Murakami, K. "Existence of a Sharply Defined Maximum Relaxation Time for Monodisperse Polystyrene” J. Polym. Sci. 1959, XL, 443-456.

53. Luap, C.; Müller, C.; Schweizer, T.; Venerus, D. C. "Simultaneous stress and birefringence measurements during uniaxial elongation of polystyrene melts with narrow molecular weight distribution" Rheol. Acta 2005, 45, 83-91. 
Only For Use in Table of Contents

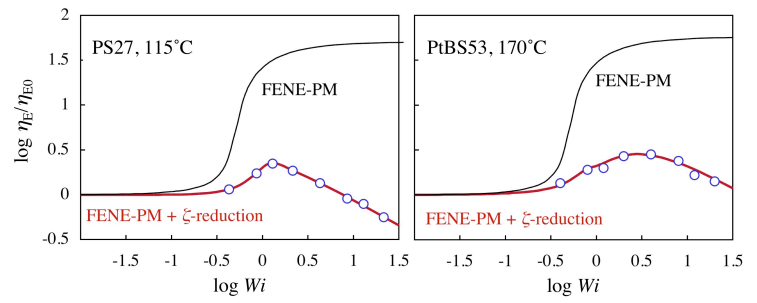

